\title{
Espaces de suites réelles complètement métrisables
}

\author{
par \\ Pierre Casevitz (Caen)
}

\begin{abstract}
Let $X$ be an hereditary subspace of the Polish space $\mathbb{R}^{\omega}$ of real sequences, i.e. a subspace such that $\left[x=\left(x_{n}\right)_{n} \in X\right.$ and $\left.\forall n,\left|y_{n}\right| \leq\left|x_{n}\right|\right] \Rightarrow y=\left(y_{n}\right)_{n} \in X$. Does $X$ admit a complete metric compatible with its vector structure? We have two results:

- If such an $X$ has a complete metric $\delta$, there exists a unique pair $(E, F)$ of hereditary subspaces with $E \subseteq X \subseteq F,(E, \delta)$ complete separable, and $F$ complete maximal in a strong sense. On $E$ and $F$, the metrics have a simple form, and the spaces $E$ are Borel $\left(\boldsymbol{\Pi}_{3}^{0}\right.$ or $\left.\boldsymbol{\Sigma}_{2}^{0}\right)$ in $\mathbb{R}^{\omega}$. In particular, if $X$ is separable, then $X=E$.

- If $X$ is an hereditary space, analytic as a subset of $\mathbb{R}^{\omega}$, we can find a subspace of $X$ strongly isomorphic to the space $c_{00}$ of finite sequences, or we can find a pair $(E, F)$ and a metric with the same properties around $X$. If $X$ is $\Sigma_{3}^{0}$ in $\mathbb{R}^{\omega}$, we get a complete trichotomy describing the possible topologies of $X$, which makes precise a result of $[\mathrm{C}]$, but for general $X$ 's, there are examples of various situations.
\end{abstract}

Le problème traité est l'étude de certains sous-espaces héréditaires, c'està-dire clos quand on réduit en valeur absolue les composantes d'un de leurs éléments, de l'espace polonais des suites de réels. Lors d'un travail connexe $[\mathrm{C}]$, parti de conjectures sur les relations d'équivalences boréliennes, on a obtenu la "dichotomie" suivante :

Si $X$ est un sous-espace héréditaire de $\mathbb{R}^{\omega}$, analytique comme partie de $\mathbb{R}^{\omega}$, alors on est dans un des deux cas suivants :

- $c_{00} \leq_{\operatorname{lin}} X$ ou $\ell_{\infty} \leq_{\operatorname{lin}} X$.

- $X$ est polonisable (comme espace vectoriel).

(Précisons les notations : $c_{00}$ et $\ell_{\infty}$ désignent l'espace des suites finies et l'espace des suites bornées ; $E \leq_{\operatorname{lin}} F$ s'il y a une application linéaire continue injective $l: \mathbb{R}^{\omega} \rightarrow \mathbb{R}^{\omega}$ telle que $l^{-1}(F)=E$.)

2000 Mathematics Subject Classification: Primary 06F20, 46A19, 46A45, 54D55; Secondary 03E15, 54E52.

Key words and phrases: complete metric vector spaces, complete metric, dichotomy theorems. 
Ce résultat suggère qu'il y a deux raisons différentes de ne pas être polonisable :

- la première c'est de ne pas être complètement métrisable (cas $c_{00}$ $\leq_{\operatorname{lin}} X$ : on peut trouver une famille dans $X$ dont toute combinaison linéaire infinie soit hors de $X$, même si on prend des coefficients $\rightarrow 0$ );

- la seconde c'est de ne pas pouvoir être rendu séparable (cas $\ell_{\infty} \leq \operatorname{lin} X$ : on peut trouver un sous-espace de $X$ complet mais pas séparable).

Dans l'idée de progresser vers une "trichotomie complète", on étudie ici les sous-espaces héréditaires de $\mathbb{R}^{\omega}$ et ceux qui sont complètement métrisables. On obtient alors les résultats suivants, plus généraux que ceux de [C], dans la mesure où, pour (1) et (2), on ne fait pas d'hypothèses sur la complexité topologique des espaces étudiés :

(1) Les sous-espaces polonisables (comme espaces vectoriels topologiques) ont une forme "canonique" : Si $X$ est polonisable, sa topologie polonaise est unique, et une distance compatible $d_{X}$ peut être définie par un procédé canonique, qui fournit aussi un espace complètement métrisable $X^{\max }$ le contenant, dont la topologie restreinte à $X$ redonne celle de $X$, et qui est maximal avec cette propriété. De plus une distance complète compatible sur $X^{\max }$ est la plus petite distance monotone sur $\mathbb{R}^{\omega}$ égale à $d_{X}$ sur $X$.

(2) Soit $Y$ un espace héréditaire complètement métrisable; alors on peut lui associer canoniquement une distance compatible complète $d_{Y}$ sur $Y$, et un unique espace polonais héréditaire $X$ tel que $X \subseteq Y \subseteq X^{\max }$ et que $d_{Y}$ soit complète sur $X$.

(3) Si $Y$ est un sous-espace analytique héréditaire de $\mathbb{R}^{\omega}$, et si $c_{00} \mathbb{L}_{\operatorname{lin}} Y$, on a un unique $X$ polonisable tel que $X \subseteq Y \subseteq X^{\max }$.

Conjecture. Ce résultat semble promettre une précision ultérieure pour la "trichotomie" espérée : ou bien $Y$ sera complètement métrisable, ou bien $c_{00} \leq_{\operatorname{lin}} Y$; éventuellement, s'il s'avère que "complètement métrisable" est trop fort, on peut remplacer par des propriétés moins fortes, comme de pouvoir être munie d'une topologie compatible avec la structure vectorielle et qui en fasse un espace de Baire. On prouve toutefois que la trichotomie est vraie pour les espaces simples du point de vue descriptif, $\boldsymbol{\Sigma}_{3}^{0}$ en fait.

(4) On a des sous-espaces analytiques héréditaires $Y$ de $\mathbb{R}^{\omega}$, munis de $X$ polonisables (uniques) tels que $X \subseteq Y \subseteq X^{\max }$, et tels que $c_{00} \leq_{\operatorname{lin}} Y$, et on en a munis d'une distance complète $d_{Y}$ compatible avec la structure vectorielle telle que $d_{Y}$ ne soit pas la restriction à $Y$ des distances de $X^{\text {max }}$ (et donc pour ces distances $Y$ n'est pas un fermé de $X^{\max }$ ).

La première partie est consacrée aux définitions. 
Ensuite, on étudie d'abord les $X$ polonisables (c'est l'essentiel du paragraphe 2), et on en déduit qu'ils ont une forme bien spécifique, à savoir qu'on peut leur associer une fonction sous-additive, définie sur $\mathbb{R}^{\omega}$ tout entier, ayant des propriétés des normes vectorielles et des sous-mesures dans $\omega$. On appellera "évaluations" de telles fonctions. Ce sont les résultats (2.1.5) et (2.2.3). C'est en fait la première partie du résultat nommé (1) ci-dessus, l'évaluation fournissant la distance canonique évoquée. Au paragraphe 2.3, on applique ces résultats pour étudier la structure des espaces complètement métrisables; on en déduit les propriétés de $X^{\max }$ dans le résultat (1), et le résultat (2).

Enfin la dichotomie générale (3) et les exemples et contre-exemples (4) sont les objets du paragraphe 4 .

Remarques. 1) Partout dans le texte, les allusions à des topologies construites ou à construire sur des espaces vectoriels, comme " $X$ est complètement métrisable", sous-entendent des topologies compatibles avec la structure vectorielle sous-jacente (sinon la distance discrète conviendrait partout). Dans un même ordre d'idées, on sous-entendra toujours que les topologies considérés sont plus fine que la topologie induite par la topologie produit usuelle sur les suites de réels, qui est la topologie de la convergence simple des fonctions de $\omega$ dans $\mathbb{R}$. Cela signifie qu'on préservera la structure d'espace de suites des espaces étudiés, même quand on change la topologie.

2) Pour les méthodes utilisées aux sections 2 et 3, certaines idées générales sont inspirées d'un travail de S. Solecki [So], effectué dans un contexte différent, celui des idéaux sur les entiers naturels (Solecki étudiait la possibilité d'être polonisables comme groupes, mais pas d'être juste complètement métrisables pour ces idéaux).

3) Pour les résultats essentiels sur les topologies polonaises, on se reportera à $[\mathrm{K}]$. Pour une étude des espaces vectoriels ordonnés, on pourra se reporter à $[\mathrm{Sc}]$.

\section{DÉFINITIONS}

1.1. Structure de $\mathbb{R}^{\omega} \cdot \mathbb{R}^{\omega}$ est muni de sa topologie polonaise usuelle, produit de la topologie de $\mathbb{R}$, pour laquelle les $W_{N}$ constituent un système fondamental de voisinages de $0=(0,0,0, \ldots)$ où

$$
W_{N}=\left\{x \in \mathbb{R}^{\omega}|\forall n<N,| x(n) \mid<1 /(N+1)\right\},
$$

et qui correspond à la métrique invariante complète suivante :

$$
d_{\omega}(x, y)=\sum_{n} \frac{\inf (1,|x(n)-y(n)|)}{2^{n}} .
$$


On désignera par l'indice ou l'exposant (pour l'adhérence par exemple) $\omega$ ce qui est relatif à la topologie usuelle de $\mathbb{R}^{\omega}$, notée elle-même $\tau_{\omega}$, ainsi qu'aux topologies induites par $\tau_{\omega}$ sur les parties de $\mathbb{R}^{\omega}$.

On notera $u_{p}$ l'élément de $\mathbb{R}^{\omega}$ dont la $(p+1)$-ème composante (c'est-à-dire $\left.u_{p}(p)\right)$ est égale à 1 et dont les autres composantes sont nulles.

Si $A \subseteq \omega$, on associe à tout $x \in \mathbb{R}^{\omega}$ les suites suivantes :

$$
\pi_{A}(x)=\left((x(k))_{k \in A},(0)_{k \notin A}\right)
$$

est la suite de $\mathbb{R}^{\omega}$ ayant comme composantes celles de $x$ pour les indices qui sont dans $A$ et 0 pour les autres indices, et

$$
\varepsilon_{A}(x)=x-\pi_{A}(x)=\pi_{\omega-A}(x) .
$$

En particulier on a

$$
\begin{aligned}
& \pi_{n}(x)=(x(0), x(1), \ldots, x(n-1), 0,0,0, \ldots), \\
& \varepsilon_{n}(x)=(0,0, \ldots, 0, x(n), x(n+1), \ldots) .
\end{aligned}
$$

Par ailleurs on identifiera couramment une $A$-suite de réels, c'est-à-dire une suite indexée par les éléments de $A$, avec la suite de $\mathbb{R}^{\omega}$ obtenue en complétant par des 0 les composantes manquantes. On considérera donc que $\mathbb{R}^{A} \subset \mathbb{R}^{\omega}$.

Enfin on notera $\mathbb{D}=\bigcup_{n} \mathbb{Q}^{n}$, ensemble des suites finies à composantes rationnelles, qui est une partie dénombrable dense canonique de $\mathbb{R}^{\omega}$.

1.2. Préordre et espaces héréditaires. On définit sur $\mathbb{R}^{\omega}$ le préordre suivant :

$$
x \leq_{\omega} y \Leftrightarrow \forall n \in \omega,|x(n)| \leq|y(n)| .
$$

On note aussi $|x|=(|x(n)|)_{n}$ la suite des valeurs absolues des composantes de $x$. Ainsi on a

$$
\left(x \leq_{\omega} y \text { et } y \leq_{\omega} x\right) \Leftrightarrow|x|=|y|, \quad x \leq_{\omega} y \Leftrightarrow|x| \leq_{\omega}|y| .
$$

Notons que ce préordre devient un ordre quand on le restreint à $\left(\mathbb{R}^{+}\right)^{\omega}$ et que l'application $x \mapsto|x|$ est croissante. On a enfin

$$
\begin{aligned}
& \left(x \leq_{\omega} y \text { et } x^{\prime} \leq_{\omega} y^{\prime}\right) \Rightarrow x+x^{\prime} \leq_{\omega}|y|+\left|y^{\prime}\right|, \\
& \left(x \leq_{\omega} y \text { et }|\lambda| \leq|\mu|\right) \Rightarrow \lambda x \leq_{\omega} \mu y .
\end{aligned}
$$

On utilisera aussi les notations suivantes :

$$
\begin{aligned}
& x \vee y=(\sup (|x(0)|,|y(0)|), \sup (|x(1)|,|y(1)|), \sup (|x(2)|,|y(2)|), \ldots), \\
& x \wedge y=(\inf (|x(0)|,|y(0)|), \inf (|x(1)|,|y(1)|), \inf (|x(2)|,|y(2)|), \ldots) .
\end{aligned}
$$

Définition (1.2.1). Une partie $X$ de $\mathbb{R}^{\omega}$ est dite héréditaire si elle est "close par suites $\leq_{\omega}$-plus petites", c'est-à-dire si $\left(x \in X\right.$ et $\left.y \leq_{\omega} x\right) \Rightarrow$ $y \in X$. 
On va s'intéresser dans ce qui suit aux sous-espaces vectoriels de $\mathbb{R}^{\omega}$ qui sont héréditaires, qu'on nomme souvent "idéaux" de $\mathbb{R}^{\omega}$ (cf. [Sc]). Pour se borner aux cas significatifs on n'étudiera que les espaces libres :

DÉfinition (1.2.2). Un sous-espace $E$ de $\mathbb{R}^{\omega}$ est libre s'il n'est nul sur aucune composante, c'est-à-dire

$$
\forall n \in \omega, \exists x \in E, \quad x(n) \neq 0 .
$$

Désormais, sauf mention expresse, les espaces considérés seront supposés libres. En effet, si $X$ n'est pas libre, ou bien il est inclus dans un $\mathbb{R}^{n}$ (et tout est beaucoup plus simple !), ou bien, à un isomorphisme près, composante par composante, entre $\mathbb{R}^{\omega}$ et un $\mathbb{R}^{A}$ avec $A$ infini, il est isomorphe à un espace libre.

Remarque (1.2.3). Si $E$ est un sous-espace héréditaire de $\mathbb{R}^{\omega}$, alors $E$ est libre si et seulement si $c_{00} \subseteq E$.

On pose aussi, définition qui sera utile dans la suite :

DÉfinition (1.2.4). Si $x \in \mathbb{R}^{\omega}$, on note $C[x]$ l'ensemble des $y$ tels que $y \leq_{\omega} x$ et $C[x]^{+}$l'ensemble des $y$ tels que $y \leq_{\omega} x$ et que chaque composante $y(n)$ soit de même signe que $x(n)$.

La propriété suivante est claire sur les définitions :

Propriété (1.2.5). $C[x]$ et $C[x]^{+}$sont des compacts de $\mathbb{R}^{\omega}$. De plus $C[x]$ est héréditaire.

Pour comparer les sous-espaces héréditaires, on va utiliser la notion suivante :

DÉfinition (1.2.6). Soient $E$ et $F$ deux sous-espaces héréditaires de $\mathbb{R}^{\omega}$.

(a) On note $E \leq_{\operatorname{lin}} F$ s'il existe une application $f: \mathbb{R}^{\omega} \rightarrow \mathbb{R}^{\omega}$ linéaire continue injective telle que $f^{-1}(F)=E$.

(b) Si on peut choisir $f$ croissante pour $\leq_{\omega}$, on note $E \leq_{\text {lin }}^{+} F$.

Pratiquement on utilisera cette définition comme suit : si on réussit à prouver que $c_{00} \leq_{\operatorname{lin}} E$, alors $E$ n'est pas complètement métrisable, sinon $c_{00}$ le serait, ce qui est impossible (on peut toujours trouver des $t_{k}$ tels que $d\left(t_{k} u_{k}, 0\right)<2^{-k}$, auquel cas $\sum_{k} t_{k} u_{k}$ converge pour $\left.d\right)$.

\section{ESPACES HÉRÉDITAIRES POLONISABLES}

On va maintenant s'intéresser aux topologies propres dont on peut doter un espace héréditaire $E$.

Pour étudier les espaces héréditaires qui seront complètement métrisables, on va introduire deux définitions. 
La première précise la compatibilité de la topologie propre d'un espace héréditaire et de l'ordre $\leq_{\omega}$ :

DÉfinition (2.0.1). Un espace héréditaire $E$ est localement héréditaire s'il a un système fondamental de voisinages de 0 qui sont des parties héréditaires de $E$ (et donc de $\mathbb{R}^{\omega}$ ).

En fait on verra que tout espace héréditaire complètement métrisable est forcément localement héréditaire.

Un outil-clef est la notion d'évaluation, analogue des sous-mesures pour les idéaux de $\omega$ et des normes observées pour les $\ell_{p}$, ou $c_{0}$, ou tous les Banach.

DÉfinition (2.0.2). Une évaluation sur $\mathbb{R}^{\omega}$ est une application $\varphi: \mathbb{R}^{\omega}$ $\rightarrow \overline{\mathbb{R}^{+}}=[0 ;+\infty]$ vérifiant les conditions suivantes :

$\operatorname{Ev}_{1} \varphi$ est positive : $\varphi(x)=0 \Leftrightarrow x=0$;

$\operatorname{Ev}_{2} \varphi$ est sous-additive : $\forall x, y, \quad \varphi(x+y) \leq \varphi(x)+\varphi(y)$;

$\mathrm{Ev}_{3} \varphi$ est croissante : $\forall x, y, \quad x \leq_{\omega} y \Rightarrow \varphi(x) \leq \varphi(y)$;

$\mathrm{Ev}_{4} \varphi$ est "sous-homogène" : $\forall x \in \mathbb{R}^{\omega}, \alpha \geq 1, \quad \varphi(\alpha x) \leq \alpha \varphi(x)$.

$\operatorname{Ev}_{5} \varphi$ est semi-continue inférieurement $:$ si $\left.\left.r \geq 0, \varphi^{-1}(] r ;+\infty\right]\right)$ est un $\tau_{\omega}$-ouvert.

$\mathrm{Ev}_{6} \varphi$ est compatible avec les topologies polonaises des sous-espaces finis $\mathbb{R}^{n}$ : sa restriction à chacun de ces espaces est continue.

REmarques. (a) Ev 5 est équivalent à l'assertion suivante :

$\mathrm{Ev}_{5}^{\prime} \quad$ pour toute suite $x_{n}$ qui $\tau_{\omega}$-converge vers une limite $x$, on a

$$
\liminf _{n} \varphi\left(x_{n}\right) \geq \varphi(x) .
$$

(b) $\varphi$ est "libre" : $\forall n, \varphi\left(u_{n}\right)<+\infty$.

Preuve. D'après $\mathrm{Ev}_{6}$ on a, pour chaque $n$, un $t<1$ tel que $\varphi\left(t u_{n}\right)<1$ et donc $\varphi\left(u_{n}\right) \leq(1 / t) \varphi\left(t u_{n}\right)<+\infty$.

On dit que $\varphi$ est finie si $\varphi(x)<+\infty$ pour tout $x$.

On dira que deux évaluations $\varphi$ et $\psi$ sont équivalentes si on a

$$
\begin{aligned}
& \forall \varepsilon>0, \exists \eta>0, \forall x, \quad \varphi(x)<\eta \Rightarrow \psi(x)<\varepsilon, \\
& \forall \varepsilon>0, \exists \eta>0, \forall x, \quad \psi(x)<\eta \Rightarrow \varphi(x)<\varepsilon .
\end{aligned}
$$

Alors toute $\varphi$ est clairement équivalente à une évaluation finie : inf $(1, \varphi)$.

2.1. Sous-espaces engendrés par une évaluation. Les espaces engendrés par une évaluation ont été étudiés dans $[\mathrm{C}]$. On rappelle ici les résultats obtenus. On notera $d_{\varphi}(x, y)=\varphi(x-y)$ sur $\mathbb{R}^{\omega}$. 
Propriété (2.1.1). $d_{\varphi}$ définit une topologie de groupe additif topologique plus fine que $\tau_{\omega}$ sur $\mathbb{R}^{\omega}$, qui est compatible avec la structure vectorielle d'un sous-espace $E$ de $\mathbb{R}^{\omega}$ si et seulement si

$$
\forall x \in E, \quad \lim _{t \rightarrow 0, t \in \mathbb{R}} \varphi(t x)=0
$$

et ceci est vérifié pour $E=c_{00}$. Enfin $d_{\varphi}$ est complète.

DÉfinition (2.1.2). Fin $(\varphi)$ est l'ensemble des $x$ tels que $\lim _{\lambda \rightarrow 0} \varphi(\lambda x)$ $=0 . \operatorname{Exh}(\varphi)$ est l'adhérence de $c_{00}$ pour $d_{\varphi}$.

REMARqUe (2.1.3). Ces espaces ne changent pas si on remplace $\varphi$ par une évaluation équivalente et les distances engendrées sont équivalentes; de plus $\varphi$ y est finie, qu'elle le soit ou non sur $\mathbb{R}^{\omega}$.

PropriÉté (2.1.4). Fin $(\varphi)$ est un sous-espace héréditaire et $d_{\varphi}$ est complète sur $\operatorname{Fin}(\varphi)$, et compatible avec sa structure vectorielle. $\operatorname{Exh}(\varphi)$ est un sous-espace héréditaire de $\operatorname{Fin}(\varphi)$ et $d_{\varphi}$ est séparable complète sur $\operatorname{Exh}(\varphi)$; $\mathbb{D}$ est dénombrable dense dans $\operatorname{Exh}(\varphi)$ et

$$
x \in \operatorname{Exh}(\varphi) \Leftrightarrow \lim _{n} \varphi\left(\varepsilon_{n}(x)\right)=0 .
$$

\subsection{Evaluations associées à un espace héréditaire polonisable.} On va montrer la réciproque de (2.1) : les espaces polonisables sont tous engendré par des évaluations.

Dans ce paragraphe $E$ désignera un sous-espace héréditaire de $\mathbb{R}^{\omega}$ polonisable et $\tau$ la topologie propre d'espace vectoriel polonais $E$. Dans $[\mathrm{C}]$ il a été démontré avec une hypothèse sur la complexité descriptive de $E$ que $E$ est de la forme $\operatorname{Exh}(\varphi)$ pour une certaine $\varphi$, mais la preuve donnée ici a l'avantage d'être constructive à partir de n'importe quelle métrique séparable complète sur $E$, ce qui donne un résultat plus précis. De plus on n'utilise pas la complexité de $E$ dans $\mathbb{R}^{\omega}$. Seule hypothèse, comme toujours : $\tau \supseteq \tau_{\omega}^{E}$. On notera aussi $d$ une distance choisie sur $E$ pour être complète, compatible avec $\tau$, invariante pour l'addition et vérifiant la propriété exigée dans la proposition ci-dessous :

Proposition (2.2.1). Si $V$ est un espace vectoriel métrique et $\delta$ une distance invariante pour l'addition et compatible avec la topologie de $V$, alors il y a une distance $d$ sur $V$ invariante pour l'addition, équivalente $\grave{a} \delta$ et vérifiant de plus:

$$
\forall \lambda \geq 1, \forall x, y \in V, \quad d(\lambda x, \lambda y) \leq \lambda d(x, y) .
$$

De plus si $V$ est complètement métrisable, $d$ peut être choisie complète.

Preuve. La dernière assertion est une conséquence des autres puisque dans un groupe complètement métrisable, toute distance invariante des deux côtés est complète. 
On connaît ce résultat quand il s'agit de groupes polonisables (cf. $[\mathrm{K}]$, $\S 9-\mathrm{A}$, p. 58) : pour toute distance $d_{1}$ invariante à gauche ou à droite sur un tel groupe, la distance $D(x, y)=d_{1}(x, y)+d_{1}\left(x^{-1}, y^{-1}\right)$ est complète, donc si $d_{1}$ est bi-invariante, elle est complète.

Or dans un groupe complètement métrisable $G$, si $d_{1}$ est invariante à gauche, la distance $D$ associée, qui est de toute façon équivalente à $d_{1}$, sera complète sur tout sous-groupe fermé séparable de $G$; en particulier si $\left(x_{n}\right)_{n}$ est $D$-Cauchy, le sous-groupe fermé engendré par les $x_{n}$ est séparable, donc $D$ y est complète et la suite converge dedans, donc dans $G$. On a donc aussi que toute distance bi-invariante est complète et que si le groupe est commutatif, toute distance invariante est complète.

Soient $V, \delta$ comme dans l'énoncé (rappelons que l'existence d'une métrique invariante pour l'addition est aussi un fait général pour les groupes métriques, cf. $[\mathrm{K}])$. Posons $\nu(x)=\delta(0, x)$. L'invariance et l'inégalité triangulaire de $\delta$ prouvent que :

(i) $\nu(x) \geq 0$ et $(\nu(x)=0 \Leftrightarrow x=0)$;

(ii) $\nu(x+y) \leq \nu(x)+\nu(y)$;

(iii) $\nu(-x)=\nu(x)$.

Réciproquement si une fonction $n$ vérifie (i)-(iii), $d(x, y)=n(x-y)$ est une distance invariante de $V$ qui sera équivalente à $\delta$ si et seulement si $n$ et $\nu$ sont équivalentes, au sens que toute $n$-boule $B=\{x \mid n(x)<r\}$ centrée en 0 contient une $\nu$-boule centrée en 0 et réciproquement.

Posons $n(x)=\sup \{\nu(t x) / t \mid t \geq 1\}$. Premièrement, par (ii) on a $\nu(p x) \leq$ $p \nu(x)$ pour tout entier $p$ et tout $x$. La distance $\delta$ étant compatible avec la topologie de $V$, la fonction $t \mapsto \delta(0, t x)=\nu(t x)$ est continue de $\mathbb{R}$ dans $\mathbb{R}$ et donc l'image de $[0 ; 1]$ est un compact $\left[0 ; a_{x}\right]$. On en déduit que pour tout $t \geq 1$, on a

$$
\nu(t x) \leq[t] \nu(x)+\nu((t-[t]) x) \leq[t] \nu(x)+a_{x},
$$

d'où l'on tire

$$
\frac{\nu(t x)}{t} \leq \frac{[t]}{t} \nu(x)+\frac{a_{x}}{t} \leq \nu(x)+a_{x}
$$

et donc $n(x) \leq \nu(x)+a_{x}<+\infty$.

Par ailleurs on a bien $n(x) \geq 0$ et comme $\nu(x)=\nu(1 x) / 1 \leq n(x)$, on a aussi $n(x)=0 \Leftrightarrow x=0$; donc $n$ vérifie la condition (i).

Comme

$$
\frac{\nu(t(x+y))}{t} \leq \frac{\nu(t x)}{t}+\frac{\nu(t y)}{t} \leq n(x)+n(y)
$$

pour tout $t \geq 1$, (ii) est vraie pour $n$.

On a $\{\nu(t x) / t \mid t \geq 1\}=\{\nu(t(-x)) / t \mid t \geq 1\}$ puisque $\nu(t(-x))=\nu(-t x)$ $=\nu(t x)$ pour tous $t, x$, donc on a aussi (iii). 
Ainsi $n$ définit bien sur $V$ une métrique invariante. On a aussi, pour $\lambda \geq 1$

$$
\begin{aligned}
n(\lambda x) & =\sup \{\nu(t \lambda x) / t \mid t \geq 1\}=\lambda \sup \{\nu(t \lambda x) /(t \lambda) \mid t \geq 1\} \\
& =\lambda \sup \{\nu(t x) / t \mid t \geq \lambda\} \leq \lambda \sup \{\nu(t x) / t \mid t \geq 1\}=\lambda n(x) .
\end{aligned}
$$

Reste à prouver que $d$ et $\delta$ sont équivalentes. Or $\nu \leq n$, donc une $\nu$-boule centrée en 0 contient forcément la $n$-boule de même rayon centrée en 0 .

Soit réciproquement la $n$-boule $B=\{x \mid n(x)<r\}$; comme tout espace vectoriel topologique possède un système fondamental de voisinages de 0 équilibrés, il y en a un (pour $\delta$ ), appelons-le $U$, inclus dans la $\nu$-boule centrée en 0 et de rayon $r / 2$, donc il y a un $\left.r^{\prime} \in\right] 0 ; r / 2\left[\right.$ tel que la $\left\{x \mid \nu(x)<r^{\prime}\right\} \subseteq U$. On a donc

$$
\nu(x)<r^{\prime} \text { et } 0 \leq t \leq 1 \Rightarrow \nu(t x)<r / 2
$$

en particulier pour $\nu(x)<r^{\prime}$ le nombre $a_{x}$ défini plus haut est $\leq r / 2$, donc d'après l'inégalité déjà établie, $n(x) \leq \nu(x)+a_{x}<r^{\prime}+r / 2<r$; la $\nu$-boule de rayon $r^{\prime}$ centrée en 0 est contenue dans $B$, ce qui termine la preuve de la proposition.

On peut donc sans perdre de généralité supposer que $E$ est muni d'une distance $d$ invariante complète vérifiant la condition ci-dessus, qu'on appellera encore "sous-homogénéité". On supposera de plus, sauf mention expresse du contraire, que $d \leq 1$, et là encore on ne perd rien puisque le changement de $d$ par $\inf (1, d)$ préserve les conditions exigées.

On va maintenant définir une évaluation sur $\mathbb{R}^{\omega}$ qui permettra de retrouver la topologie de $E$.

DÉFinition (2.2.2). On appelle évaluation associée à $(E, d)$ la fonction $\varphi$ définie sur $\mathbb{R}^{\omega}$, à valeurs dans $[0 ; 1]$ (ou $\overline{\mathbb{R}^{+}}$si on n'a pas supposé que $d \leq 1$ ), telle que pour tout $x$,

$$
\varphi(x)=\sup \left\{d(0, y) \mid y \in E, y \leq_{\omega} x\right\} .
$$

Le principal résultat sur les sous-espaces héréditaires polonisables est le suivant, dont la démonstration occupe le reste de ce paragraphe.

Proposition (2.2.3). (1) $\varphi$ est une évaluation: elle vérifie les propriétés $\mathrm{Ev}_{1} \grave{a} \mathrm{Ev}_{6}$.

(2) $E$ et $\operatorname{Exh}(\varphi)$ coïncident et la topologie $\tau$ coïncide avec celle induite $\operatorname{par} \varphi$.

Corollaire (2.2.4). Tout espace héréditaire qui est polonisable est localement héréditaire. De plus c'est un sous-ensemble $\Pi_{3}^{0}$ de $\left(\mathbb{R}^{\omega}, \tau_{\omega}\right)$, et en fait les autres résultats démontrés sur de telles espaces s'appliquent, notamment il est fermé, $\boldsymbol{\Sigma}_{2}^{0}$-complet ou $\boldsymbol{\Pi}_{3}^{0}$-complet. 
Preuve. La première assertion vient de ce que si $d$ et $\varphi$ engendrent la même topologie, un système fondamental de voisinages de 0 est la suite des boules $\left\{x \in E \mid \varphi(x)<2^{-n}\right\}$, qui sont clairement héréditaires. La deuxième vient de ce que $\operatorname{Exh}(\varphi)$ est $\boldsymbol{\Pi}_{3}^{0}$ puisque

$$
x \in \operatorname{Exh}(\varphi) \Leftrightarrow \forall n, \exists p, \quad \varphi\left(\varepsilon_{p}(x)\right) \leq 2^{-n}
$$

et que d'après la semi-continuité de $\varphi, \varphi\left(\varepsilon_{p}(x)\right) \leq 2^{-n}$ est une condition fermée.

Les autres assertions ont été établies dans $[\mathrm{C}]$ pour les $E$ héréditaires polonisables qui sont des parties analytiques de $\mathbb{R}^{\omega}$. Comme un espace héréditaire polonisable est forcément $\Pi_{3}^{0}$, ces résultats s'appliquent.

La proposition va être établie en une série d'étapes.

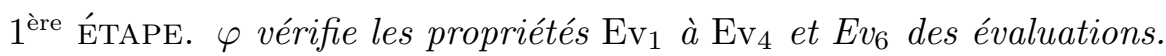

- $\operatorname{Ev}_{1}$ ( $\varphi$ est positive) : En effet, si $x \neq 0$, il y a un $k$ tel que $0 \neq$ $x(k) u_{k} \leq_{\omega} x$; or $u_{k} \in E$, donc $\varphi(x) \geq d\left(0, x(k) u_{k}\right)>0$.

- $\operatorname{Ev}_{2}$ ( $\varphi$ est sous-additive) : En effet, soit $z \leq_{\omega} x+y, z \in E$. Montrons qu'on a forcément

$$
d(0, z) \leq \varphi(x)+\varphi(y) .
$$

On a $|z(k)| \leq|x(k)+y(k)| \leq|x(k)|+|y(k)|$. Pour tout $k$ tel que $|x(k)|+$ $|y(k)|>0$, posons

$$
a(k)=\frac{|x(k)|}{|x(k)|+|y(k)|} z(k), \quad b(k)=\frac{|y(k)|}{|x(k)|+|y(k)|} z(k) ;
$$

si $|x(k)|+|y(k)|=0$ on pose $a(k)=b(k)=0=z(k)$. On aura donc pour tout $k$,

$$
|a(k)| \leq|(z \wedge x)(k)|, \quad|b(k)| \leq|(z \wedge y)(k)|, \quad a(k)+b(k)=z(k) ;
$$

donc $a, b \in E$ et $d(0, a) \leq \varphi(x)$ et $d(0, b) \leq \varphi(y)$ entraînent $d(0, z)=d(0, a+b) \leq d(0, a)+d(a, a+b)=d(0, a)+d(0, b) \leq \varphi(x)+\varphi(y)$.

- $\operatorname{Ev}_{3}$ ( $\varphi$ est croissante) : En effet, si $x \leq_{\omega} x^{\prime}$ alors

$$
\left\{d(0, y) \mid y \in E, y \leq_{\omega} x\right\} \subseteq\left\{d(0, y) \mid y \in E, y \leq_{\omega} x^{\prime}\right\} .
$$

- $\operatorname{Ev}_{4}$ ( $\varphi$ est sous-homogène) : Soit $t>1$. Montrons que pour tout $a$, $a>t \varphi(x) \Rightarrow a \geq \varphi(t x)$. Supposons donc $a>t \varphi(x)$, et prenons un $y \in E$ tel que $y \leq_{\omega} t x$. Alors $(1 / t) y \leq_{\omega} x$ et $(1 / t) y \in E$, donc $d(0,(1 / t) y) \leq \varphi(x)<$ $(1 / t) a$ et

$$
d(0, y)=d(0, t(1 / t) y) \leq t d(0,(1 / t) y)<a .
$$

- $\operatorname{Ev}_{6}$ ( $\varphi$ est compatible avec les topologies polonaises des sous-espaces finis $\mathbb{R}^{n}$ ) : Il suffit de montrer que la restriction de $\varphi$ à chaque droite $\mathbb{R} u_{n}$ est 
continue en 0 : cela entraînera que $\varphi\left(x_{0}, \ldots, x_{n-1}, 0,0, \ldots\right) \leq \sum_{k<n} \varphi\left(x_{k} u_{k}\right)$ est aussi continue en 0 si on munit $\mathbb{R}^{n}$ de la norme $\sup _{k<n}|x(k)|$ par exemple.

Or la fonction $t \mapsto \varphi\left(t u_{n}\right)$ tend bien vers 0 en 0 : en effet, soit $\varepsilon>0$; comme $d$ est compatible avec la structure vectorielle de $E \supseteq \mathbb{R} u_{n}$, on a $r$ tel que $|t|<r \Rightarrow d\left(0, t u_{n}\right)<\varepsilon$. Mais comme $x \leq_{\omega} t u_{n} \Leftrightarrow\left(x=s u_{n}\right.$ et $|s| \leq|t|)$, on a en fait $|t|<r \Rightarrow\left(x \leq_{\omega} t u_{n} \Rightarrow d(0, x)<\varepsilon\right)$ et finalement $|t|<r \Rightarrow \varphi\left(t u_{n}\right) \leq \varepsilon$, ce qui achève la démonstration de $\mathrm{Ev}_{6}$.

$2^{\text {ème }}$ ÉTAPE. $\varphi$ est semi-continue inférieurement.

Cette assertion se démontre moyennant le lemme suivant :

Lemme (2.2.5). Pour tout $y \in E$, on a $\lim _{n} d\left(0, \varepsilon_{n}(y)\right)=0$.

Admettons ce résultat; soit $r>0$; prouvons que l'ensemble

$$
\Omega=\{x \mid \varphi(x)>r\}
$$

est un $\tau_{\omega}$-ouvert. Si $x \in \Omega$, on a donc $y \in E$ tel que $y \leq_{\omega} x$ et $r<d(0, y)$. D'après le lemme on a $\lim _{n} d\left(0, \varepsilon_{n}(y)\right)=\lim _{n} d\left(\pi_{n}(y), y\right)=0$.

Il existe donc un $n_{0}$ tel que $d\left(0, \pi_{n_{0}}(y)\right)>r$. En particulier, $\pi_{n_{0}}(y) \leq_{\omega}$ $\pi_{n_{0}}(x)$, donc $\varphi\left(\pi_{n_{0}}(x)\right) \geq \varphi\left(\pi_{n_{0}}(y)\right) \geq d\left(0, \pi_{n_{0}}(y)\right)>r$ et d'après $\operatorname{Ev}_{6}$ il y a un $\mathbb{R}^{n_{0}}$-ouvert $U$ autour de $\pi_{n_{0}}(x)$ sur lequel $\varphi$ est $>r$. Par suite si $x^{\prime} \in U \times \mathbb{R}^{\omega-n_{0}}$, on a $\pi_{n_{0}}\left(x^{\prime}\right) \in U$, donc $\varphi\left(x^{\prime}\right) \geq \varphi\left(\pi_{n_{0}}\left(x^{\prime}\right)\right)>r$, d'où $U \times \mathbb{R}^{\omega-n_{0}} \subseteq \Omega$, ce qui prouve bien que $\Omega$ est ouvert.

Preuve du lemme (2.2.5). On va montrer que la suite $\left(\varepsilon_{n}(y)\right)_{n}$ est $d$-Cauchy; comme $d$ est complète, elle aura une limite, et comme $\tau$ est plus fine que $\tau_{\omega}$, cette limite sera forcément celle qu'elle a dans $\tau_{\omega}$, c'est-à-dire 0 .

Soient $\varepsilon>0$ et $b$ tels que $6 b<\varepsilon$. On se place dans l'ensemble $C[y]^{+}$qui est $\tau_{\omega}$-compact, donc $\tau$-fermé; les topologies induites sont donc toutes deux polonaises, celle induite par $\tau_{\omega}$ étant moins fine que l'autre.

Une conséquence est qu'elles engendrent les mêmes boréliens sur $C[y]^{+}$, en particulier les intersections des $\tau$-boréliens de $E$ avec $C[y]^{+}$sont des boréliens de $C[y]^{+}$pour $\tau_{\omega}^{C[y]^{+}}$.

FAIT. Il y a un $N$, un $A \in c_{00} \cap C[y]^{+}$et un $z \in E$ tels que le d-ouvert $\{t \in E \mid d(z, t)<b\} \cap C[y]^{+}$de $C[y]^{+}$soit comaigre au sens de la topologie induite par $\tau_{\omega}$ dans $\left(A+W_{N}\right) \cap C[y]^{+}$.

En effet, comme $\left(C[y]^{+}, \tau\right)$ est polonais il y a une suite dense $\left(z_{n}\right)_{n}$ et donc les boules $\left\{t \in E \mid d\left(z_{n}, t\right)<b\right\}$ recouvrent $C[y]^{+}$. D'après ce qui précède, ces boules sont pour $\tau_{\omega}^{C[y]^{+}}$des boréliens et donc ont la propriété de Baire. Il s'ensuit qu'il y a un ouvert $U$ de $\tau_{\omega}^{C[y]^{+}}$et un $z$ pour lesquels $\{t \in E \mid d(z, t)<b\} \cap C[y]^{+}$soit $\tau_{\omega}^{C[y]^{+}}$-comaigre dans $U$. Comme cela reste vrai pour tout sous-ouvert de $U$, on peut supposer qu'on a $r>0, P$ et $A(0) \in[0 ; y(0)], \ldots, A(P-1) \in[0 ; y(P-1)]$ tels que $U$ contienne l'ouvert 
$V=\{t|\forall k<P| t,(k)-A(k) \mid<r\} \cap C[y]^{+}$. On pose comme d'habitude $A(k)=0$ si $k \geq P$.

On a alors, si $N$ est tel que $1 /(N+1)<r$ et $N>P$, la configuration décrite dans le fait.

Pour $\varepsilon$ et donc $b$ donnés, $N, A$ et $z$ sont donc fixés par le fait ci-dessus.

Choisissons maintenant $M>N$; on a

$$
\left(A+W_{M}\right) \cap C[y]^{+} \subseteq\left(A+W_{N}\right) \cap C[y]^{+}=V .
$$

Comme conséquence de $\mathrm{Ev}_{6}, d$ est équivalente à la métrique usuelle sur $\mathbb{R}^{M}$, et on a aussi $c>0$ tel que

$$
(\forall k<M,|t(k)|<c) \Rightarrow d(0,(t(0), \ldots, t(M-1)))<b .
$$

Posons $V^{\prime}=\left(A+W_{M}\right) \cap\{t|\forall k<M, \quad| t(k)-A(k) \mid<c / 2\} \cap C[y]^{+}$. Alors $V^{\prime}$ est un sous-ouvert (pour $\tau_{\omega}^{C[y]^{+}}$) de $V$, donc tout comaigre dans $V$ l'est dans $V^{\prime}$.

Considérons maintenant $f: C[y]^{+} \rightarrow C[y]^{+}$définie par $f\left((x(k))_{k}\right)=$ $\left((x(k))_{k<M},(y(k)-x(k))_{k \geq M}\right) ; f$ est une involution continue pour $\tau_{\omega}^{C[y]^{+}}$ et est donc un homéomorphisme. De plus $f\left(V^{\prime}\right)=V^{\prime}$.

Il s'ensuit que $f\left(\{t \in E \mid d(z, t)<b\} \cap C[y]^{+}\right)$est comaigre dans $f\left(V^{\prime}\right)$ $=V^{\prime}$, et que

$$
f\left(\{t \in E \mid d(z, t)<b\} \cap C[y]^{+}\right) \cap\{t \in E \mid d(z, t)<b\} \cap C[y]^{+}
$$

est comaigre dans $V^{\prime}$, donc non vide.

Soit $\xi$ un de ses points. Alors $d(\xi, z)<b$ et

$$
\begin{aligned}
\xi \in f(\{t \in E \mid d(z, t)<b\} & \left.\cap C[y]^{+}\right) \\
& \Leftrightarrow f(\xi) \in\{t \in E \mid d(z, t)<b\} \cap C[y]^{+},
\end{aligned}
$$

d'où $d(f(\xi), z)<b$. Donc

$$
d(2 z, \xi+f(\xi)) \leq d(2 z, z+\xi)+d(z+\xi, f(\xi)+\xi)<2 b .
$$

Or

$$
f(\xi)+\xi=(2 \xi(0), \ldots, 2 \xi(M-1), y(M), y(M+1), \ldots)=2 \pi_{M}(\xi)+\varepsilon_{M}(y) .
$$

Comme $\xi \in V^{\prime}$, on a $|2(\xi(k)-A(k))|<c$ pour $k<M$, d'où par le choix de $c$,

$$
d\left(0,2 \pi_{M}(\xi-A)\right)<b .
$$

Finalement

$$
\begin{aligned}
& d\left(2 z-2 A, \varepsilon_{M}(y)\right) \\
& \quad \leq d\left(2 z-2 A, 2 \pi_{M}(\xi)+\varepsilon_{M}(y)-2 A\right)+d\left(2 \pi_{M}(\xi)+\varepsilon_{M}(y)-2 A, \varepsilon_{M}(y)\right) \\
& \quad=d\left(2 z, 2 \pi_{M}(\xi)+\varepsilon_{M}(y)\right)+d\left(2 \pi_{M}(\xi)-2 A, 0\right) \\
& \quad=d\left(2 z, 2 \pi_{M}(\xi)+\varepsilon_{M}(y)\right)+d\left(2 \pi_{M}(\xi-A), 0\right) \quad \operatorname{car} A \in \mathbb{R}^{N} \\
& \quad=d(2 z, \xi+f(\xi))+d\left(2 \pi_{M}(\xi-A), 0\right)<2 b+b=3 b .
\end{aligned}
$$


On a donc prouvé que pour $M>N$ tous les points $\varepsilon_{M}(y)$ sont dans la $d$-boule de centre $2(z-A)$ et de rayon $3 b$. Il s'ensuit que deux de ces points sont distants au plus de $2 \cdot 3 b=6 b<\varepsilon$.

Comme $N$ ne dépend que de $\varepsilon$, ceci prouve bien que la suite $\left(\varepsilon_{M}(y)\right)_{M}$ est de Cauchy.

$3^{\text {ème }}$ ÉTAPE. $E \subseteq \operatorname{Exh}(\varphi)$ et si $x \in E$, sur $C[x]$ les topologies induites par $\tau$ et $\tau_{\omega}$ coïncident avec celle induite par $\varphi$.

Commençons par examiner la fin de l'assertion : soit $x \in E$. On sait que $\tau \supseteq \tau_{\omega}$, il suffit donc de montrer l'inclusion inverse sur $C[x]$. On va montrer qu'une suite $\tau_{\omega}$-convergente est aussi $\tau$-convergente.

FAIT. Une suite $\left(y_{n}\right)_{n}$ convergeant vers 0 dans $\tau_{\omega}^{C[x]^{+}}$converge vers 0 pour $d$.

Supposons que $d\left(0, y_{n}\right) \nrightarrow 0$. Alors il y a un $r>0$ et une infinité de $n$ tels que $d\left(0, y_{n}\right)>r$. On suppose qu'on n'a gardé que ces $n$ et que $d\left(0, y_{n}\right)>r$ pour tout $n$. Construisons par recurrence deux suites : $n_{0}=0<n_{1}<\ldots$ et $k_{0}=0<k_{1}<\ldots$ telles que pour tout $p$ on ait

$$
d\left(0, \pi_{\left[k_{p}, k_{p+1}[\right.}\left(y_{n_{p}}\right)\right)>r / 2
$$

de la manière suivante : d'après le lemme (2.2.5), $\pi_{k}\left(y_{0}\right) \rightarrow y_{0}$ pour $d$, donc il y a bien un $k_{1}$ tel que $d\left(0, \pi_{k_{1}}\left(y_{0}\right)\right)>r>r / 2$. Maintenant comme $y_{n} \rightarrow 0$ dans $\tau_{\omega}$, et que les topologies induites coïncident sur $\mathbb{R}^{k_{1}}$, on aura un $n_{1}$ tel que

$d\left(0, \pi_{k_{1}}\left(y_{n_{1}}\right)\right)<r / 2 \quad$ et $\quad d\left(0, \varepsilon_{k_{1}}\left(y_{n_{1}}\right)\right) \geq d\left(0, y_{n_{1}}\right)-d\left(0, \pi_{k_{1}}\left(y_{n_{1}}\right)\right)>r / 2$.

En refaisant pour $\varepsilon_{k_{1}}\left(y_{n_{1}}\right)$ le raisonnement fait pour trouver $k_{1}$, on trouve $k_{2}>k_{1}$ tel que $d\left(0, \pi_{k_{2}}\left(\varepsilon_{k_{1}}\left(y_{n_{1}}\right)\right)\right)=d\left(0, \pi_{\left[k_{1}, k_{2}[\right.}\left(y_{1}\right)\right)>r / 2$. Puis on trouve $n_{2}>n_{1}$ tel que $d\left(0, \pi_{k_{2}}\left(y_{n_{2}}\right)\right)<r / 2$ puis $k_{3}>k_{2}$ tel que $d\left(0, \pi_{\left[k_{2}, k_{3}\right.}\left(y_{2}\right)\right)>$ $r / 2$ et ainsi de suite. On construit ainsi nos suites $\left(k_{p}\right)_{p}$ et $\left(n_{p}\right)_{p}$ et on pose $v_{p}=\pi_{\left[k_{p}, k_{p+1}[\right.}\left(y_{n_{p}}\right)$.

Dans $\tau_{\omega}$, comme les supports des $v_{p}$ sont disjoints, leur somme $s=\sum_{p} v_{p}$ est définie. Chaque composante de $s$ est majorée par la composante correspondante d'un $y_{n}$, donc par la composante correspondante de $x$ puisque $y_{n} \in C[x]$. Donc $s \leq_{\omega} x$ et $s \in E$. Le lemme (2.2.5) montre que $d\left(0, \varepsilon_{k}(s)\right)$ $\rightarrow 0$ or $d\left(\varepsilon_{k_{p}}(s), \varepsilon_{k_{p+1}}(s)\right)=d\left(0, \pi_{\left[k_{p}, k_{p+1}[\right.}\left(y_{n_{p}}\right)\right)>r / 2$, donc cette suite n'est même pas de Cauchy, une contradiction.

Le fait est donc établi.

On en déduit que tout $d$-voisinage de 0 dans $C[x]$ contient un $W_{N} \cap C[x]$ car sinon on pourrait extraire une suite $y_{N} \in W_{N} \cap C[x]$ qui ne converge pas vers 0 pour $d$. 
Soit maintenant une suite $\left(y_{n}\right)_{n}$ dans $C[x]$ qui converge vers $y$ pour $\tau_{\omega}$; alors on a

$$
d\left(y, y_{n}\right) \leq d\left(\pi_{k}(y), \pi_{k}\left(y_{n}\right)\right)+d\left(0, \varepsilon_{k}(y)\right)+d\left(0, \varepsilon_{k}\left(y_{n}\right)\right) \quad \text { pour tous } n, k .
$$

Soit un $\varepsilon>0$ et prenons un $K$ tel que $d(0, z)<\varepsilon / 3$ si $z \in W_{K} \cap C[x]$. Alors $z \leq_{\omega} x \Rightarrow \varepsilon_{K}(z) \in W_{K} \cap C[x]$, donc

$$
d\left(0, \varepsilon_{K}(y)\right)<\varepsilon / 3, \quad d\left(0, \varepsilon_{K}\left(y_{n}\right)\right)<\varepsilon / 3 \quad \text { pour tout } n .
$$

Comme $y_{n} \rightarrow y$ dans $\tau_{\omega}$, d'après $\mathrm{Ev}_{6}$ il $\mathrm{y}$ a un rang $n_{0}$ à partir duquel $d\left(\pi_{K}(y), \pi_{K}\left(y_{n}\right)\right)<\varepsilon / 3$, donc pour $n \geq n_{0}$ on a bien $d\left(y_{n}, y\right)<\varepsilon$ et finalement $d\left(y_{n}, y\right) \rightarrow 0$.

Maintenant la topologie induite par $\varphi$ est encore la même car on pourrait refaire tout le raisonnement en remplaçant $E \operatorname{par} \operatorname{Exh}(\varphi)$ et $d$ par la distance associée à $\varphi$. La seule chose à vérifier est que $x \in \operatorname{Exh}(\varphi)$ si $x \in E$; mais c'est une conséquence de ce qui précède : on a bien $\varphi\left(\varepsilon_{n}(x)\right) \rightarrow 0$ car si $\varepsilon>0$ est donné, il y a un $N$ tel que $y \in W_{N} \cap C[x] \Rightarrow d(0, y)<\varepsilon$ et en particulier $y \leq_{\omega} \varepsilon_{N}(x) \Rightarrow d(0, y)<\varepsilon$, d'où $\varphi\left(\varepsilon_{N}(x)\right) \leq \varepsilon$.

Ainsi l'étape 3 est complète.

$4^{\text {ème }}$ ÉTAPE. $E=\operatorname{Exh}(\varphi)$ et les topologies induites par $d$ et $\varphi$ coïncident sur ces espaces.

On a vu dans la $3^{\text {ème }}$ étape que $E \subseteq \operatorname{Exh}(\varphi)$ et comme les topologies induites sur $C[x]$ coïncident pour $x \in E$ avec celle induite par $\tau_{\omega}, \mathbb{D}$ est dense dans $E$. Considérons l'application identique $i$ de $(E, d)$ dans $\left(E, d_{\varphi}\right)$. Comme $\mathbb{D}$ est dense dans $\left(\operatorname{Exh}(\varphi), d_{\varphi}\right)$, il est dense dans $\left(E, d_{\varphi}\right) .\left(E, d_{\varphi}\right)$ est donc un groupe séparable pour l'addition, et comme $i$ est borélienne et $(E, d)$ polonais, $i$ est en fait continue (pour ces critères de continuité d'applications entre groupes topologiques, voir le théorème de Pettis et ses conséquences, par exemple dans $[\mathrm{K}], \S 9-\mathrm{C}$, th. 9 et 10 , p. 61$)$.

Dans le sens inverse, $d(x, y) \leq \varphi(x-y)$, donc $i^{-1}$ est continue et finalement $i$ est un homéomorphisme. Comme on a des distances bi-invariantes pour l'addition, $i$ est uniformément bicontinue, et l'image $i(E)=E$ de l'espace complet $(E, d)$ par $i$ est un sous-espace complet $\left(E, d_{\varphi}\right)$ de $\left(\operatorname{Exh}(\varphi), d_{\varphi}\right)$, c'est-à-dire que $E$ est $d_{\varphi}$-fermé dans $\operatorname{Exh}(\varphi)$. Comme il y a une partie dense commune, ces deux espaces sont bien égaux et comme $i$ est un homéomorphisme, leurs topologies coïncident.

La démonstration est donc achevée.

On cherche maintenant à appliquer les résultats précédents pour étudier les espaces héréditaires complètement métrisables.

2.3. Sous-espaces héréditaires complètement métrisables. On a pour l'instant des résultats sur les espaces polonisables. On va maintenant 
essayer de les étendre à une classe d'espaces plus large, complètement métrisables mais pas forcément séparables. Cette classe comprend notamment les espaces de Fréchet, mais ceux-ci sont localement convexes et la "bonne" notion relative aux sous-espaces héréditaires n'est pas la locale convexité mais la locale hérédité (2.0.1).

En fait, on va voir que le résultat du (2.2.4) qui dit que tout espace héréditaire polonisable est localement héréditaire se généralise à tous les espaces complètement métrisables : il s'ensuivra qu'un tel espace apparaît comme un sous-espace héréditaire d'un espace $\operatorname{Fin}(\varphi)$ muni de sa propre distance complète qui vérifiera une certaine compatibilité avec $\varphi$.

Dans ce paragraphe, $X$ désignera un espace héréditaire de $\mathbb{R}^{\omega}$ muni d'une distance complète invariante pour l'addition (voir preuve de (2.2.1)) $d_{X}$ qui définit sa topologie d'espace vectoriel métrique $\tau_{X}$. Par ailleurs on notera $\tau_{\infty}$ la topologie d'espace de Banach de $\ell_{\infty}$, que définit sa norme usuelle $\|\cdot\|_{\infty}$.

Comme toujours, $\tau_{X} \supseteq \tau_{\omega}^{X}$.

Définition-Notation (2.3.1). Si $x, y \in \mathbb{R}^{\omega}$, on note $x * y$ l'élément de $\mathbb{R}^{\omega}$ dont la composante d'indice $k$ est $x(k) y(k)$.

Proposition (2.3.2). (i) Si $\Lambda \in \ell_{\infty}$ et $x \in X$, on a $\Lambda * x \in X$.

(ii) Quel que soit $\Lambda \in \ell_{\infty}$, l'application $x \mapsto \Lambda * x$ est linéaire continue de $\left(X, \tau_{X}\right)$ dans lui-même.

(iii) Quel que soit $x \in X$, l'application $\Lambda \mapsto \Lambda * x$ est linéaire continue de $\left(\ell_{\infty}, \tau_{\infty}\right)$ dans $\left(X, \tau_{X}\right)$.

Corollaire (2.3.3). (i) Pour toute partie héréditaire $H \subseteq X$, l'adhérence $\bar{H}^{\tau_{X}}$ de $H$ pour $d_{X}$ est héréditaire.

(ii) Il y a un unique sous-espace héréditaire de $X$ sur lequel $\tau_{X}$ induit une topologie polonaise, et c'est l'adhérence de $\mathbb{D}$ dans $\tau_{X}$.

Preuve de la proposition. (i) est clair puisque $X$ est héréditaire et qu'on a $\Lambda * x \leq_{\omega}\|\Lambda\|_{\infty} x$.

(ii) La linéarité est évidente. Reste la continuité.

D'après le théorème du graphe fermé pour les espaces vectoriels complètement métrisables (cf. [B], ch. I, cor. 5, p. 19), il suffit de montrer que le graphe de la fonction considérée est fermé. Or la fonction $x \mapsto \Lambda * x$ est continue de $\left(\mathbb{R}^{\omega}, \tau_{\omega}\right)$ dans lui-même, et donc de $\left(X, \tau_{\omega}^{X}\right)$ dans lui-même. Son graphe est donc fermé pour $\tau_{\omega}^{X} \times \tau_{\omega}^{X}$, et comme $\tau_{X}$ est plus fine que $\tau_{\omega}^{X}$, il reste fermé pour $\tau_{X} \times \tau_{X}$.

(iii) C'est exactement le même argument que pour (ii), appliqué au graphe de la fonction considérée dans $\tau_{\infty} \times \tau_{X}$.

Preuve du corollaire. (i) Soit $x \in \bar{H}^{\tau_{X}}$; on a donc une suite $\left(x_{n}\right)_{n}$ de points de $H$ qui tend vers $x$ dans $\tau_{X}$. Soit $y \leq_{\omega} x$. Cela signifie que $|y(k)| \leq$ $|x(k)|$ pour tout $k$ ou, de manière équivalente, qu'on peut trouver une suite 
de nombres $\Lambda(k) \in[-1 ; 1]$ tels que $y(k)=\Lambda(k) x(k)$, c'est-à-dire que $\|\Lambda\|_{\infty} \leq$ 1 et $y=\Lambda * x$.

D'après la proposition $\Lambda * x_{n}$ tend vers $\Lambda * \lim x_{n}=\Lambda * x=y$; or $\Lambda * x_{n} \leq_{\omega} x_{n} \in H$, donc par hérédité $\Lambda * x_{n} \in H$, ce qui prouve bien que $y \in \bar{H}^{\tau} \bar{X}$.

$\bar{H}^{\tau_{X}}$ est donc bien héréditaire.

(ii) On sait par (2.2.4) que si la restriction de $d_{X}$ à un sous-espace héréditaire $Y \subseteq X$ induit une topologie polonaise, $\mathbb{D}$ y est dense. De plus $\left(d_{X}\right)_{\mid Y}$ étant invariante est complète sur $Y, Y$ est $\tau_{X}$-fermé. Il s'ensuit que l'adhérence $\overline{\mathbb{D}}^{\tau_{X}}$ de $\mathbb{D}$ est égale à $Y$.

Il suffit de vérifier que $\overline{\mathbb{D}}^{\tau X}$ est bien un sous-espace vectoriel héréditaire.

Comme $\tau_{X}$ induit sur $\mathbb{R}^{n}$ une topologie polonaise compatible avec la structure vectorielle, il s'agit en fait de la topologie usuelle. Donc $\mathbb{Q}^{n}$ est $\tau_{X^{-}}$dense dans $\mathbb{R}^{n}$, et en en prenant la réunion pour tous les $n, \mathbb{D}$ est $\tau_{X^{-}}$ dense dans $c_{00}$ et $\overline{\mathbb{D}}^{\tau_{X}}=\bar{c}_{00}^{\tau_{X}}$. Enfin $\bar{c}_{00}^{\tau_{X}}$ est bien un sous-espace vectoriel comme adhérence d'un sous-espace, c'est un ensemble héréditaire d'après le (i). Le corollaire est donc prouvé.

Ainsi tout espace complètement métrisable contient un de nos espaces polonisables héréditaires. On notera $E_{X}=\overline{\mathbb{D}}^{\tau_{X}}$ cet espace. D'après (2.2.1) on peut supposer que $d_{X}$ est invariante complète et sous-homogène et alors on a suivant (2.2.2) que $\varphi(x)=\sup \left\{d_{X}(0, y) \mid y \leq_{\omega} x\right.$ et $\left.y \in E_{X}\right\}$ est une évaluation, qu'on appellera évaluation associée $\grave{a}\left(X, d_{X}\right)$, telle que $E_{X}=$ $\operatorname{Exh}(\varphi)$ et $d_{X}$ et $d_{\varphi}$ sont équivalentes sur $E_{X}$.

Deux questions se posent sur les rapports entre $\varphi$ et $d_{X}$ :

- $X$ est-il forcément un sous-espace de $\operatorname{Fin}(\varphi)$ (sinon cela signifie que $\varphi$ n'est même pas compatible avec la structure vectorielle de $X$ ) ?

- Si oui, $d_{X}$ et $\varphi$ sont-elles équivalentes dans $X$ ?

Pour résoudre le premier problème, il faut étudier plus en détail l'application $(\Lambda, x) \mapsto \Lambda * x$ déjà évoquée.

DÉfinition (2.3.4). On notera $\mathcal{L}$ l'espace vectoriel des applications linéaires continues de $\left(\ell_{\infty},\|\cdot\|_{\infty}\right)$ dans $\left(X, d_{X}\right)$ et pour tout $x \in X$, on notera $g_{x}$ l'application $\Lambda \mapsto \Lambda * x$.

Proposition (2.3.5). (i) $\mathcal{L}$ est complètement métrisable et une distance invariante sous-homogène complète est donnée par

$$
d_{\mathcal{L}}(0, f)=\sup \left\{d_{X}(0, f(\Lambda)) \mid\|\Lambda\|_{\infty} \leq 1\right\} .
$$

(ii) L'application $x \mapsto g_{x}$ est un isomorphisme linéaire de $\left(X, \tau_{X}\right)$ sur un sous-espace fermé de $\left(\mathcal{L}, d_{\mathcal{L}}\right)$. 
Preuve. (i) Si $f \in \mathcal{L}$, posons $d^{\prime}(f)=\sup \left\{d_{X}(0, f(\Lambda)) \mid\|\Lambda\|_{\infty} \leq 1\right\}$. Comme $f$ est continue, il y a $\alpha$ tel que $\|\Lambda\|_{\infty} \leq \alpha \Rightarrow d_{X}(0, f(\Lambda)) \leq 1$; alors

$$
\begin{aligned}
&\|\Lambda\|_{\infty} \leq 1 \Rightarrow\left\|\alpha \frac{\Lambda}{\|\Lambda\|_{\infty}}\right\|_{\infty} \leq \alpha \Rightarrow d_{X}\left(0, f\left(\alpha \frac{\Lambda}{\|\Lambda\|_{\infty}}\right)\right) \leq 1 \\
& \Rightarrow d_{X}(0, f(\Lambda))=d_{X}\left(0, f\left(\frac{\|\Lambda\|_{\infty}}{\alpha} \cdot \frac{\alpha}{\|\Lambda\|_{\infty}} \cdot \Lambda\right)\right) \\
& \leq d_{X}\left(0, f\left(\alpha \frac{\Lambda}{\|\Lambda\|_{\infty}}\right)\right) \cdot \sup \left(1, \frac{\|\Lambda\|_{\infty}}{\alpha}\right) \leq \sup \left(1, \frac{1}{\alpha}\right) .
\end{aligned}
$$

Ainsi $d^{\prime}$ est finie même si $d_{X}$ n'est pas bornée.

On a bien pour $f, g \in \mathcal{L}$ et $\|\Lambda\|_{\infty} \leq 1$,

$$
d_{X}((f+g)(\Lambda), 0) \leq d_{X}(f(\Lambda), 0)+d_{X}(g(\Lambda), 0) \leq d^{\prime}(f)+d^{\prime}(g) ;
$$

donc $d^{\prime}(f+g) \leq d^{\prime}(f)+d^{\prime}(g)$. On voit de même $d^{\prime}(-f)=d^{\prime}(f), d^{\prime}(\lambda f) \leq$ $\sup (1,|\lambda|) d^{\prime}(f)$ et comme pour $f \in \mathcal{L}$ et $\Lambda \in \ell_{\infty}$ on a $d_{X}\left(f\left(\Lambda /\|\Lambda\|_{\infty}\right), 0\right) \leq$ $d^{\prime}(f)$ on aura aussi

$$
d^{\prime}(f)=0 \Rightarrow \forall \Lambda, \quad f(\Lambda)=0 \Rightarrow f=0 .
$$

Pour montrer que $d^{\prime}(f-g)$ est une distance compatible avec la structure vectorielle de $\mathcal{L}$ il suffit de prouver qu'on a $\lim _{t \rightarrow 0, t \in \mathbb{R}} d^{\prime}(t f)=0$ pour tout $f \in \mathcal{L}$. Or une telle $f$ étant continue, on a

$$
\forall \varepsilon>0, \exists \eta>0, \quad\|\Lambda\|_{\infty} \leq \eta \Rightarrow d_{X}(0, f(\Lambda)) \leq \varepsilon .
$$

Donc si $|t| \leq \eta$, on a pour tout $\Lambda$ tel que $\|\Lambda\|_{\infty} \leq 1$,

$$
d_{X}(0,(t f)(\Lambda))=d_{X}(0, f(t \Lambda)) \text { et }\|t \Lambda\|_{\infty} \leq \eta \Rightarrow d_{X}((t f)(\Lambda), 0) \leq \varepsilon .
$$

Donc $d^{\prime}(t f) \leq \varepsilon$. Ceci prouve bien que $\lim _{t \rightarrow 0, t \in \mathbb{R}} d^{\prime}(t f)=0$.

Enfin reste à montrer que $d_{\mathcal{L}}(f, g)=d^{\prime}(f-g)$ est complète. Soit $\left(f_{n}\right)_{n}$ de Cauchy. Comme

$$
\begin{aligned}
d_{X}\left(f_{n}(\Lambda), f_{p}(\Lambda)\right) & \leq \sup \left(1,\|\Lambda\|_{\infty}\right) d_{X}\left(f_{n}\left(\frac{\Lambda}{\|\Lambda\|_{\infty}}\right), f_{p}\left(\frac{\Lambda}{\|\Lambda\|_{\infty}}\right)\right) \\
& \leq \sup \left(1,\|\Lambda\|_{\infty}\right) d_{\mathcal{L}}\left(f_{n}, f_{p}\right),
\end{aligned}
$$

la suite $\left(f_{n}(\Lambda)\right)_{n}$ est de Cauchy dans $\left(X, d_{X}\right)$ pour tout $\Lambda$ et $\left(f_{n}\right)_{n}$ converge simplement vers une fonction $f$ de $\ell_{\infty}$ dans $X$, qui est linéaire comme on le voit en faisant $n \rightarrow \infty$ dans $f_{n}(\alpha \Lambda+\beta M)=\alpha f_{n}(\Lambda)+\beta f_{n}(M)$.

Soit $\varepsilon>0$. On a $n$ tel que pour $p, q \geq n$ on ait $d_{\mathcal{L}}\left(f_{q}, f_{p}\right)<\varepsilon$. Pour $\|\Lambda\|_{\infty} \leq 1$ on a donc

$$
p, q \geq n \Rightarrow d_{X}\left(f_{q}(\Lambda), f_{p}(\Lambda)\right)<\varepsilon
$$

donc si on fait $q$ fixe et $p \rightarrow \infty$ il vient

$$
\forall \Lambda,\|\Lambda\|_{\infty} \leq 1 \Rightarrow d_{X}\left(f_{q}(\Lambda), f(\Lambda)\right) \leq \varepsilon .
$$


Comme $f_{q}$ est continue, il y a un $r \leq 1$ tel que $\|\Lambda\|_{\infty} \leq r \Rightarrow d_{X}\left(f_{q}(\Lambda), 0\right)$ $<\varepsilon$, donc

$$
\|\Lambda\|_{\infty} \leq r \Rightarrow d_{X}(f(\Lambda), 0) \leq d_{X}\left(f_{q}(\Lambda), f(\Lambda)\right)+d_{X}\left(f_{q}(\Lambda), 0\right)<2 \varepsilon .
$$

Comme on pourrait trouver de tels $q, r$ pour chaque $\varepsilon, f$ est bien continue en 0 , donc linéaire continue.

Reprenons maintenant notre $\varepsilon$ fixé et le $n$ qu'on a trouvé tel que pour $q \geq n$,

$$
\forall \Lambda, \quad\|\Lambda\|_{\infty} \leq 1 \Rightarrow d_{X}\left(f_{q}(\Lambda), f(\Lambda)\right) \leq \varepsilon .
$$

Ceci signifie bien que $d_{\mathcal{L}}\left(f_{q}, f\right) \leq \varepsilon$ si $q \geq n$. D'où $\lim _{n} d_{\mathcal{L}}\left(f_{n}, f\right)=0$ et $f$ est bien la $d_{\mathcal{L}}$-limite de $\left(f_{n}\right)_{n}$, ce qui achève la preuve de (i).

(ii) $x \mapsto g_{x}$ est linéaire, il suffit donc, pour montrer qu'elle est continue, de prouver que son graphe est fermé, ou encore que pour toute suite $\left(x_{n}\right)_{n}$ convergeant vers 0 , si $\left(g_{x_{n}}\right)_{n}$ a une limite dans $\mathcal{L}$, c'est 0 .

Soit $\left(x_{n}\right)_{n}$ une telle suite. D'après $(2.3 .2), g_{x_{n}}(\Lambda)=\Lambda * x_{n}$ tend vers 0 dans $\left(X, \tau_{X}\right)$ pour tout $\Lambda$. D'après (i), une suite $\left(g_{x_{n}}\right)_{n}$ qui converge pour $d_{\mathcal{L}}$ tend simplement vers sa limite. Donc si $\left(g_{x_{n}}\right)_{n}$ a une limite, c'est 0 . La fonction $x \mapsto g_{x}$ est donc bien continue.

Elle est injective car si $g_{x}=0$, on a $g_{x}\left(u_{p}\right)=0 \Rightarrow x(p)=0$, d'où $x=0$.

Enfin notons $I$ l'élément de $\ell_{\infty}$ dont toutes les composantes sont égales à 1 , de sorte que $g_{x}(I)=x$. On a clairement

$$
\|I\|_{\infty} \leq 1 \Rightarrow d_{X}\left(g_{y}(I), g_{x}(I)\right)=d_{X}(y, x) \leq d_{\mathcal{L}}\left(g_{y}, g_{x}\right) .
$$

Donc si $\left(g_{x_{n}}\right)_{n}$ est $d_{\mathcal{L}^{-}}$-Cauchy, $\left(x_{n}\right)_{n}$ est $d_{X}$-Cauchy, donc a une limite $x \in X$ et par continuité $g_{x_{n}} \rightarrow g_{x}$ pour $d_{\mathcal{L}}$, ce qui prouve bien que $\left\{g_{x} \mid x \in X\right\}$ est fermé complet dans $\left(\mathcal{L}, d_{\mathcal{L}}\right)$. Cela suffit pour assurer que $x \mapsto g_{x}$ est bicontinue, donc est bien un isomorphisme d'espaces complètement métrisables.

Proposition (2.3.6). (i) Tout espace héréditaire $X$ complètement métrisable est localement héréditaire et admet une distance qui est invariante par translation, sous-homogène, complète et $\leq_{\omega}$-croissante.

(ii) Si $\varphi$ est une évaluation associée à $X$ et $E_{X}=\operatorname{Exh}(\varphi)$, alors

$$
E_{X} \subseteq X \subseteq \operatorname{Fin}(\varphi) .
$$

Preuve. (i) Posons $D(x)=d_{\mathcal{L}}\left(g_{x}, 0\right)$ pour $x \in X$. On a vu au (2.3.5) que, par l'isomorphisme $x \mapsto g_{x}, D(x-y)$ est une distance invariante soushomogène complète sur $X$, topologiquement équivalente à $d_{X}$. De plus

$$
\begin{aligned}
D(x) & =\sup \left\{d_{X}\left(0, g_{x}(\Lambda)\right) \mid\|\Lambda\|_{\infty} \leq 1\right\}=\sup \left\{d_{X}(0, \Lambda * x) \mid\|\Lambda\|_{\infty} \leq 1\right\} \\
& =\sup \left\{d_{X}(0, y) \mid y \leq_{\omega} x\right\},
\end{aligned}
$$

donc la fonction $D(x)$ est $\leq_{\omega}$-croissante. Pour une telle distance les boules $\{x \mid D(x)<r\}$ sont héréditaires, donc $X$ est localement héréditaire. 
(ii) Soit $x \in X$; montrons que $\varphi(t x)$ tend vers 0 . Soit $\varepsilon>0$; il y a un voisinage héréditaire $V$ de 0 dans $X$ tel que

$$
z \in V \Rightarrow d_{X}(0, z)<\varepsilon \text {. }
$$

Par continuité partielle de $(t, x) \mapsto t x$ il y a, pour chaque $x$ fixé, un $\eta$ tel que

$$
|t|<\eta \Rightarrow t x \in V
$$

Dans ce cas $y \leq_{\omega} t x \Rightarrow d(0, y)<\varepsilon$ et en particulier

$$
|t|<\eta \Rightarrow \varphi(t x)=\sup \left\{d(0, y) \mid y \in E_{X}, y \leq_{\omega} t x\right\} \leq \varepsilon .
$$

On a donc bien $x \in \operatorname{Fin}(\varphi)$.

PropriÉté (2.3.7). Soit $\left(X, d_{X}\right)$ un sous-espace héréditaire de $\mathbb{R}^{\omega}$ muni d'une distance qui en fasse un espace vectoriel métrique complet dont la topologie est plus fine que celle de la convergence simple sur les suites.

(i) $S i \varphi$ est une évaluation telle que

$$
\operatorname{Exh}(\varphi) \subseteq X \subseteq \operatorname{Fin}(\varphi)
$$

alors $\operatorname{Exh}(\varphi)=\bar{c}_{00}^{X}$.

(ii) Il existe un unique couple d'espaces héréditaires $(E, F)$ tels que $E \subseteq$ $X \subseteq F$ et qu'il existe une évaluation $\varphi$ telle que $E=\operatorname{Exh}(\varphi)$ et $F=\operatorname{Fin}(\varphi)$. Les évaluations vérifiant ces égalités sont deux à deux équivalentes.

(iii) Si Y est un sous-espace héréditaire quelconque, et $\psi, \psi^{\prime}$ deux évaluations telles que

$$
\operatorname{Exh}(\psi) \subseteq Y \subseteq \operatorname{Fin}(\psi) \text { et } \operatorname{Exh}\left(\psi^{\prime}\right) \subseteq Y \subseteq \operatorname{Fin}\left(\psi^{\prime}\right),
$$

alors $\operatorname{Exh}(\psi)=\operatorname{Exh}\left(\psi^{\prime}\right), \operatorname{Fin}(\psi)=\operatorname{Fin}\left(\psi^{\prime}\right)$, et $\psi$ et $\psi^{\prime}$ sont équivalentes.

Preuve. On suppose conformément au résultat (2.3.6) qu'on a muni $X$ d'une distance $d_{X}$ complète compatible avec l'addition et telle que $x \mapsto$ $d_{X}(0, x)$ soit $\leq_{\omega}$-croissante.

(i) On va commencer par prouver le fait suivant :

FAIT. Dans la situation de (2.3.7)(i) on a $\bar{c}_{00}^{X} \subseteq \operatorname{Exh}(\varphi)$.

On va raisonner par l'absurde : supposons qu'on trouve $x \in \bar{c}_{00}^{X}-\operatorname{Exh}(\varphi)$. Alors $x \in \bar{c}_{00}^{X}$, donc $d_{X}\left(0, \varepsilon_{n}(x)\right) \rightarrow 0$ quand $n \rightarrow \infty$.

Par ailleurs $x \notin \operatorname{Exh}(\varphi)$, donc $\varphi\left(\varepsilon_{n}(x)\right) \nrightarrow 0$ quand $n \rightarrow \infty$, et comme la suite $\left(\varphi\left(\varepsilon_{n}(x)\right)\right)_{n}$ est décroissante, cela signifie qu'il y a un $r>0$ tel que $\varphi\left(\varepsilon_{n}(x)\right)>r$ pour tout $n$, et on peut donc trouver une suite $n_{0}<n_{1}<$ $\ldots<n_{k}<\ldots$ d'entiers tels que $\varphi\left(\pi_{\left[n_{k}, n_{k+1}[\right.}(x)\right)>r$ pour tout $k$.

Soit $\xi_{k}=\pi_{\left[n_{k}, n_{k+1}[\right.}(x)$. Les $\xi_{k}$ ont des supports disjoints et comme $\xi_{k} \leq_{\omega}$ $\varepsilon_{n_{k}}(x)$, on a $d_{X}\left(0, \xi_{k}\right) \leq d_{X}\left(0, \varepsilon_{n_{k}}(x)\right) \rightarrow 0$ quand $k \rightarrow \infty$. On peut donc 
extraire une suite $v_{p}=\xi_{k_{p}}$ telle qu'on ait $d_{X}\left(0, v_{p}\right) \leq 1 / 4^{p}$ et toujours $\varphi\left(v_{p}\right)>r$.

Posons $T=\sum_{p} 2^{p} v_{p}$, qui est définit dans $\mathbb{R}^{\omega}$ car les supports des $v_{p}$ sont disjoints. Or par sous-additivité de $d_{X}$, on a

$$
d_{X}\left(0,2^{p} v_{p}\right) \leq 2^{p} d_{X}\left(0, v_{p}\right) \leq 2^{p} \frac{1}{4^{p}}=\frac{1}{2^{p}}
$$

Comme $d_{X}$ est complète, la série $\left(2^{p} v_{p}\right)_{p}$ est convergente dans $X$, ce qui signifie que $T \in X$, donc $T \in \operatorname{Fin}(\varphi)$. Mais pour $a \neq 0$, il y a forcément un $p_{0}$ tel que $\left|a 2^{p_{0}}\right| \geq 1$, d'où $v_{p_{0}} \leq_{\omega} a 2^{p_{0}} v_{p_{0}} \leq_{\omega} a T$ et $\varphi(a T) \geq \varphi\left(v_{p_{0}}\right)>r$. Ceci est une contradiction avec la définition de $\operatorname{Fin}(\varphi)$.

Si on applique le fait à $X$ on trouve $\bar{c}_{00}^{X} \subseteq \operatorname{Exh}(\varphi)$. D'après (2.3.3) et (2.3.6) il existe une évaluation $\varphi_{0}$ telle que $\operatorname{Exh}\left(\varphi_{0}\right)=\bar{c}_{00}^{X}$ et $X \subseteq \operatorname{Fin}\left(\varphi_{0}\right)$. Mais alors $Y=\operatorname{Exh}(\varphi)$ vérifie $\operatorname{Exh}\left(\varphi_{0}\right) \subseteq Y \subseteq \operatorname{Fin}\left(\varphi_{0}\right)$. On peut donc réappliquer le fait : $\bar{c}_{00}^{Y} \subseteq \operatorname{Exh}\left(\varphi_{0}\right)$. Mais $Y=\operatorname{Exh}(\varphi)$ entraîne que les suites finies sont denses dans $Y$ muni de sa topologie d'espace vectoriel métrique complet (séparable), c'est-à-dire $Y=\bar{c}_{00}^{Y}$, ce qui prouve bien que $\operatorname{Exh}(\varphi)=Y=\operatorname{Exh}\left(\varphi_{0}\right)=\bar{c}_{00}^{X}$. (i) est donc démontré.

(ii) Soient $\varphi$ et $\psi$ deux évaluations telles que $\operatorname{Exh}(\varphi)=\operatorname{Exh}(\psi)$. Elles définissent donc deux topologies forcément identiques sur $\operatorname{Exh}(\varphi)=E$. $\operatorname{En}$ particulier les distances induites définissent les mêmes voisinages d'un point de $E$ dans $E$, et donc aussi d'un point de $c_{00}$ dans $c_{00}$. On a

$$
\forall \varepsilon>0, \exists \eta>0, \forall x \in c_{00}, \quad \varphi(x)<\eta \Rightarrow \psi(x)<\varepsilon .
$$

Soit $x \in \mathbb{R}^{\omega}$. Par semi-continuité inférieure on sait que

$$
\psi(x)=\sup \left\{\psi\left(\pi_{n}(x)\right) \mid n \in \mathbb{N}\right\} .
$$

Donc si $\varphi(x)<\eta$, on a $\varphi\left(\pi_{n}(x)\right) \leq \varphi(x)<\eta$ pour chaque $n$, donc $\psi\left(\pi_{n}(x)\right)$ $<\varepsilon$, ce qui prouve $\psi(x) \leq \varepsilon$. Comme on peut intervertir les rôles de $\varphi$ et de $\psi$, on voit que les deux évaluations sont bien équivalentes, et définissent du coup le même espace Fin, ce qu'on a prouvé en supposant juste que les Exh étaient les mêmes.

Soient maintenant $X, E, F, \varphi$ donnés dans (ii) et une $\varphi_{0}$ associée à $X$ par (2.3.3). Alors $\operatorname{Exh}(\varphi)=\operatorname{Exh}\left(\varphi_{0}\right)$ par le (i) et ce qui précède prouve alors qu'on a bien $\operatorname{Fin}(\varphi)=\operatorname{Fin}\left(\varphi_{0}\right)$ et $\varphi$ et $\varphi_{0}$ équivalentes.

(iii) Posons $\mu(x)=\psi(x)+\psi^{\prime}(x)$; alors $\mu$ est une évaluation telle que $\operatorname{Exh}(\mu)=\operatorname{Exh}(\psi) \cap \operatorname{Exh}\left(\psi^{\prime}\right)$ et $\operatorname{Fin}(\mu)=\operatorname{Fin}(\psi) \cap \operatorname{Fin}\left(\psi^{\prime}\right)$. De plus on a $\operatorname{Exh}(\mu) \subseteq \operatorname{Exh}(\psi) \subseteq Y \subseteq \operatorname{Fin}(\psi) \cap \operatorname{Fin}\left(\psi^{\prime}\right)=\operatorname{Fin}(\mu)$, et on peut appliquer le (i) et le (ii) à $\varphi=\mu$ et $X=\operatorname{Exh}(\psi)$; on obtient que $\operatorname{Exh}(\psi)=\operatorname{Exh}(\mu)$ et que $\psi$ et $\mu$ sont équivalentes. On procède de même pour $\psi^{\prime}$ et on conclut facilement. 
A tout espace héréditaire complètement métrisable on associe donc un couple d'espaces héréditaires et une classe d'évaluations deux à deux équivalentes uniquement définis.

\section{LA PROPRIÉTÉ GÉNÉRALE D'ENCADREMENT}

Dans cette section, $X$ désignera un espace héréditaire de $\mathbb{R}^{\omega}$ qu'on suppose libre et analytique comme partie de $\mathbb{R}^{\omega}$.

On fera un usage fréquent du théorème suivant, dû à Talagrand $[\mathrm{T}]$ :

Si $\mathcal{I}$ est un idéal sur $\omega$ qui est libre (il contient les parties finies) et non trivial $(\omega \notin \mathcal{I})$, et si $\mathcal{I}$ est Baire-mesurable comme partie du compact $2^{\omega}$, alors il y a une partition $\left\{F_{n}\right\}_{n}$ de $\omega$ en parties finies telles que

$$
\forall B \subseteq \omega, \quad \bigcup_{n \in B} F_{n} \in \mathcal{I} \Leftrightarrow B \text { est finie. }
$$

3.1. Espaces impropres. On rappelle ici des résultats élémentaires démontrés dans $[\mathrm{C}]$. Il s'agit d'éliminer un type d'espaces héréditaires en quelque sorte "dégénérés".

DÉfinition (3.1.1). Un espace héréditaire $X$ est dit propre s'il existe un élément $x \in X$ dont les composantes sont toutes non nulles. Sinon il est dit impropre.

Proposition (3.1.2). Si $X$ est impropre, $c_{00} \leq_{\operatorname{lin}}^{+} X$.

Dans le reste de la section on supposera donc qu'on considère des espaces propres.

\subsection{La dichotomie}

ThÉORÈme (3.2.1). Si X est un sous-espace analytique héréditaire de $\mathbb{R}^{\omega}$, on a au moins une des deux assertions suivantes :

- $c_{00} \leq_{\operatorname{lin}}^{+} X ;$ ou

- Il y a une évaluation $\varphi$ telle que $\operatorname{Exh}(\varphi) \subseteq X \subseteq \operatorname{Fin}(\varphi)$.

Si la deuxième assertion est vraie, le couple $(\operatorname{Exh}(\varphi), \operatorname{Fin}(\varphi))$ est unique, conformément à (2.3.7)(iii).

La démonstration prendra toute la section 3.2. On suppose donné $X$ comme dans l'énoncé, et on suppose qu'il est propre.

DÉfinition (3.2.2). Un ensemble quelconque $A \subseteq \mathbb{R}^{\omega}$ sera dit petit s'il est inclus dans une réunion de fermés héréditaires $F_{n} \subseteq \mathbb{R}^{\omega}$ tels qu'il existe un $x \in X$ pour lequel la droite $\mathbb{R} x$ privée de l'origine ne rencontre aucun $F_{n}$ :

$$
A \subseteq \bigcup_{n} F_{n} \quad \text { et } \quad \forall \lambda \in \mathbb{R}^{*}, \forall n, \lambda x \notin F_{n} .
$$

Un ensemble héréditaire $A \subseteq \mathbb{R}^{\omega}$ sera dit grand s'il n'est pas petit. 
Propriété (3.2.3). Soit $F$ un fermé héréditaire de $\mathbb{R}^{\omega}$.

(i) Les assertions suivantes sont équivalentes:

- F est grand;

- F rencontre toute droite de $X$ en un point distinct de 0 au moins : $\forall x \in X, \exists \lambda \in \mathbb{R}^{*}, \quad \lambda x \in F$

- F vérifie

$$
\forall x \in X, \exists n \in \omega, n>0, \quad \frac{1}{n} x \in F .
$$

(ii) Si $x \in X$, et si $\mathbb{R}^{*} x \cap F=\emptyset$, on $a$

$\forall v \in\left(\mathbb{R}_{+}^{*}\right)^{\omega}, \forall m \in \omega, m>0, \forall n \in \omega, \exists p \geq n, \quad \pi_{n}(v)+\frac{1}{m} \pi_{] n, p]}(x) \notin F$.

Preuve. (i) L'équivalence des deux dernières assertions est une conséquence de l'hérédité de $F:$ si $\lambda x \in F$, on a aussi $\mu x \in F$ dès que $|\mu| \leq \lambda$.

S'il y a un $x \in X$ mettant en défaut la deuxième propriété, alors $F$ est petit en appliquant la définition avec chaque $F_{n}$ égal à $F$.

Si $F$ vérifie cette propriété, il n'est certainement pas petit puisque la définition de la petitesse fournit un $x$ comme contre-indiqué.

(ii) Si cette propriété était en défaut, il y aurait $v, m, n$ pour la contredire. Mais dans $\mathbb{R}^{\omega}$, on a $\pi_{n}(v)+m^{-1} \pi_{] n, p]}(x) \rightarrow \pi_{n}(v)+m^{-1} \varepsilon_{n}(x)$ si $p \rightarrow \infty$. $F$ étant fermé, on aurait donc $\pi_{n}(v)+m^{-1} \varepsilon_{n}(x) \in F$. Comme $v \in\left(\mathbb{R}_{+}^{*}\right)^{\omega}$, il y a un $M$ assez grand pour que $M>m$ et $M^{-1} \pi_{n}(x) \leq_{\omega} \pi_{n}(v)$. On en déduit $M^{-1} x \leq_{\omega} \pi_{n}(v)+m^{-1} \varepsilon_{n}(x)$ et donc $M^{-1} x \in F$ par hérédité, ce qui contredit l'hypothèse faite. de $\mathbb{R}^{\omega}$.

PropriÉté (3.2.4). (i) Les ensembles petits forment un idéal de parties

(ii) L'intersection de deux ensembles fermés héréditaires grands est grande.

Preuve. (i) Une sous-partie d'une partie petite reste clairement petite. Soient $A, A^{\prime}$ deux parties petites et, associés par la définition, $\left\{F_{n}\right\}_{n},\left\{F_{n}^{\prime}\right\}_{n}$ des fermés héréditaires, ainsi que $x, x^{\prime} \in X$. Alors $\bigcup_{n} F_{n} \cup \bigcup_{n} F_{n}^{\prime} \supseteq A \cup A^{\prime}$ et si $z=|x|+\left|x^{\prime}\right|$, on a $z \in X$ et pour tout $\lambda, \lambda x \leq_{\omega} \lambda z$ et $\lambda x^{\prime} \leq_{\omega} \lambda z$, donc ces $\lambda z$ ne sont sûrement dans aucun $F_{n}$ ou $F_{n}^{\prime}$, sinon par hérédité $\lambda x$ ou $\lambda x^{\prime}$ seraient dedans. Finalement les collections $F_{n}$ et $F_{n}^{\prime}$, et $z$ prouvent que $A \cup A^{\prime}$ est petit.

(ii) Un fermé héréditaire est grand s'il rencontre toute droite $\mathbb{R} x$ de $X$ en un point $\lambda x \neq 0$. Pour deux tels fermés $F$ et $F^{\prime}$, ils rencontrent $\mathbb{R} x$ en $\lambda x$ et $\lambda^{\prime} x$, et si $\mu=\inf \left(|\lambda|,\left|\lambda^{\prime}\right|\right)$, alors par hérédité $\mu x \neq 0$ est dans $F \cap F^{\prime}$.

La dichotomie va porter sur le caractère de $\sigma$-idéal ou non de cet idéal. On va d'abord se placer dans le cas où il y a une suite de fermés héréditaires 
petits dont la réunion est grande. Comme $X$ est propre, on peut donc choisir un $u \in X \cap\left(\mathbb{R}_{+}^{*}\right)^{\omega}$.

Hypothèse (3.2.5). Il y a une famille $\left\{A_{n}\right\}_{n \in \omega}$ de parties de $\mathbb{R}^{\omega}$ et des $x_{n} \in X$ tels que :

- $A_{n}$ est fermé héréditaire;

- $A_{n} \cap \mathbb{R}^{*} x_{n}=\emptyset$;

- $\forall x \in X, \bigcup_{n} A_{n} \cap \mathbb{R}^{*} x \neq \emptyset$.

Proposition (3.2.6). L'hypothèse (3.2.5) équivaut à l'assertion: "Les petits ne forment pas un $\sigma$-idéal".

Preuve. Si (3.2.5) est vraie alors elle fournit des ensembles qui témoignent que les petits ne forment pas un $\sigma$-idéal.

Si les petits ne forment pas un $\sigma$-idéal, il y a des $P_{n}$ qui sont petits et dont la réunion n'est pas petite. Soient pour chaque $n$ des $F_{n}^{k}$ et un $y_{n} \in X$ témoignant la petitesse. Si on considère les $F_{n}^{k}$, associés chacun à leur $y_{n}$, leur réunion rencontre sûrement toute droite de $X$ hors de 0 , sinon il témoigneraient de la petitesse de $\bigcup_{n} P_{n}$. Donc ils vérifient (3.2.5).

Proposition (3.2.7). Sous-l'hypothèse (3.2.5) on a $c_{00} \leq_{\operatorname{lin}}^{+} X$.

Preuve. Soient les $\left\{A_{n}\right\}_{n}$ et les $\left(x_{n}\right)_{n}$ donnés par (3.2.5). Les propriétés énoncées dans (3.2.5) ne changeant pas, grâce à l'hérédité, si on remplace chaque $x_{n}$ par $\left|x_{n}\right|+u$, on supposera que $x_{n} \in\left(\mathbb{R}_{+}^{*}\right)^{\omega}$ pour tout $n$.

On peut de plus supposer :

(P) toute union infinie de $A_{n}$ est grande.

En effet, si $A \subseteq \omega$, la propriété " $\bigcup_{n \in A} A_{n}$ est petite" s'écrit

$$
\exists x \in X, \forall p \in \omega, \forall n \in A, \quad \frac{1}{p+1} x \notin A_{n} .
$$

C'est donc une propriété analytique de $A$, ces $A$ forment donc un idéal analytique $I$ qui est visiblement libre et non trivial (l'assertion " $\omega$ n'est pas dedans" est précisément (3.2.5)), et on peut (par $[\mathrm{T}]$ ) trouver une partition de $\omega$ en ensembles finis $B_{m}$ dont seules les unions finies sont dans $I$. Si on remplace les $A_{n}$ et les $x_{n}$ par les $\bigcup_{n \in B_{m}} A_{n}=A_{m}^{\prime}$ et $x_{m}^{\prime}=\sum_{n \in B_{m}}\left|x_{n}\right|$, on obtient visiblement la propriété $(\mathrm{P})$. On supposera donc qu'elle est vérifiée, en gardant les notations de (3.2.5).

Si on choisit un des $x_{n}$, un $P \in \omega, P>0$ et un rang $N \in \omega$, on aura, d'après (3.2.3) (où on prend $v=P^{-1} u$ ), un $M>N$ tel que $P^{-1}\left(\pi_{N}(u)+\right.$ $\left.\pi_{[N, M[}\left(x_{n}\right)\right) \notin A_{n}$.

On fixe $(n, p) \mapsto\langle n, p\rangle$ une bijection $\omega^{2} \rightarrow \omega$ telle que $\langle 0,0\rangle=0$. On notera, pour chaque $(n, p),\left(n^{\prime}, p^{\prime}\right)$ l'unique couple tel que $\left\langle n^{\prime}, p^{\prime}\right\rangle=\langle n, p\rangle+1$. 
On construit alors une suite $N_{p}^{n}$ d'entiers par récurrence sur le nombre $\langle n, p\rangle$ : on part de $N_{0}^{0}=0$, et si on suppose construits les nombres $N_{p}^{n}$ pour $\langle n, p\rangle=0,1, \ldots, k$, on choisit $N_{p^{\prime}}^{n^{\prime}}$ avec $\left\langle n^{\prime}, p^{\prime}\right\rangle=k+1$ tel que

$$
\frac{1}{p+1}\left(\pi_{N_{p}^{n}}(u)+\pi_{\left[N_{p}^{n}, N_{p^{\prime}}^{n^{\prime}}\right.}\left(x_{n}\right)\right) \notin A_{n} .
$$

Avec cette construction on a donc

$$
\forall n, p, \quad \frac{1}{p+1}\left(\pi_{N_{p}^{n}}(u)+\pi_{\left[N_{p}^{n}, N_{p^{\prime}}^{n^{\prime}}\right.}\left(x_{n}\right)\right) \notin A_{n} .
$$

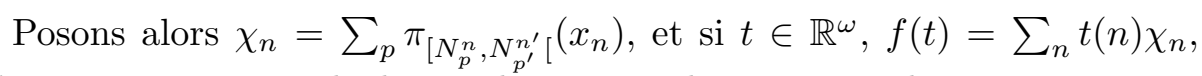
qu'on peut toujours calculer car les $\chi_{n}$ ont des supports disjoints.

On a $\chi_{n} \leq_{\omega} x_{n}$, donc $\chi_{n} \in X$, et donc une combinaison linéaire finie des $\chi_{n}$ reste dans $X$, soit $f\left(c_{00}\right) \subseteq X$.

Mais considérons $t \in \mathbb{R}^{\omega}-c_{00}$, et posons $T=|f(t)|+u$. Soit un $n$ tel que $t(n) \neq 0$ et un réel $\lambda \neq 0$. On peut trouver $P$ tel que $1 /(P+1) \leq$ $\inf (|\lambda|,|\lambda t(n)|)$. Alors on a

$$
\begin{aligned}
\frac{1}{P+1}\left(\pi_{N_{n}^{P}}(u)+\pi_{\left[N_{n}^{P}, N_{n^{\prime}}^{P^{\prime}}[\right.}\left(\chi_{n}\right)\right) & \leq_{\omega} \lambda \pi_{N_{n}^{P}}(u)+\lambda t(n) \pi_{\left[N_{n}^{P}, N_{n^{\prime}}^{P^{\prime}}[\right.}\left(\chi_{n}\right) \\
& \leq_{\omega} \lambda \pi_{N_{n^{\prime}}^{P^{\prime}}}(u+|f(t)|) \leq_{\omega} \lambda T .
\end{aligned}
$$

Comme $(P+1)^{-1}\left(\pi_{N_{n}^{P}}(u)+\pi_{\left[N_{n}^{P}, N_{n^{\prime}}^{P^{\prime}}[\right.}\left(\chi_{n}\right)\right) \notin A_{n}$ par construction, on a aussi, par hérédité, $\lambda T \notin A_{n}$. On a donc obtenu

$$
\forall n, \quad\left[t(n) \neq 0 \Rightarrow \forall \lambda \neq 0, \quad \lambda T \notin A_{n}\right] ;
$$

la réunion des $A_{n}$ pour $t(n) \neq 0$ est une réunion infinie de $A_{n}$, donc grande. La droite $\mathbb{R} T$ ne la rencontre pas hors de l'origine. Donc $T \notin X$ et du même coup $f(t) \notin X$.

Ainsi $f$ est le plongement cherché.

Hypothèse (3.2.8). Pour toute famille $\left\{P_{n}\right\}_{n \in \omega}$ de parties de $\mathbb{R}^{\omega}$ qui soient petites, il y a un $x \in X$ et des $\left\{A_{n}\right\}_{n \in \omega}$ tels que :

- $A_{n}$ est fermé héréditaire;

- $A_{n} \cap \mathbb{R}^{*} x=\emptyset$;

- $\bigcup_{n} P_{n} \subseteq \bigcup_{n} A_{n}$.

Proposition (3.2.9). L'hypothèse (3.2.8) équivaut à : Les petits forment un $\sigma$-idéal.

La proposition (3.2.9) est claire vu les définitions, et il est clair qu'une des deux assertions (3.2.5) ou (3.2.8) est vraie.

Proposition (3.2.10). Sous l'hypothèse (3.2.8), on a une évaluation $\varphi$ telle que $\operatorname{Exh}(\varphi) \subseteq X \subseteq \operatorname{Fin}(\varphi)$; s'ils existent, les espaces $\operatorname{Exh}(\varphi)$ et $\operatorname{Fin}(\varphi)$ sont uniques, et $\varphi$ est unique, modulo l'équivalence d'évaluations. 
Preuve. On va considérer la compactification $\boldsymbol{K}=[-\infty ;+\infty]^{\omega}$ de $\mathbb{R}^{\omega}$.

On étend sans difficultés les définitions du préordre, de l'hérédité, de la prise du sup ou de l'inf de deux suites, à $\boldsymbol{K}$; les opérations $\vee, \wedge$ restent continues.

On définit aussi

$$
\operatorname{Her}(A)=\left\{x \mid \exists y \in A, \quad x \leq_{\omega} y\right\} .
$$

On notera par l'indice ou l'exposant " $\boldsymbol{K}$ " les notions relatives à $\boldsymbol{K}$ et à sa topologie.

On va maintenant rappeler brièvement le rapport entre topologie et préordre dans $\boldsymbol{K}$, étudié dans $[\mathrm{C}]$.

FAIT. (i) Il y a une injection de l'espace $\mathcal{F}\left(\mathbb{R}^{\omega}\right)$ des fermés de $\mathbb{R}^{\omega}$ dans l'espace $\mathcal{K}(\boldsymbol{K})$ des compacts de $\boldsymbol{K}$. Cette injection est $F \mapsto \bar{F}^{\boldsymbol{K}}$, et un inverse à gauche est $K \mapsto K \cap \mathbb{R}^{\omega}$. De plus $F \in \mathcal{F}\left(\mathbb{R}^{\omega}\right)$ est héréditaire si et seulement si $\bar{F}^{K}$ l'est.

(ii) Si $K \in \mathcal{K}(\boldsymbol{K})$, alors $\operatorname{Her}(K) \in \mathcal{K}(\boldsymbol{K})$; de plus $K \mapsto \operatorname{Her}(K)$ est continue sur $\mathcal{K}(\boldsymbol{K})$ et l'ensemble des fermés héréditaires est un compact de $\mathcal{K}(\boldsymbol{K})$.

Preuve. Tout ceci a été démontré dans $[\mathrm{C}]$.

On définit dans $\mathcal{K}(\boldsymbol{K})$ les ensembles suivants :

- $\mathcal{H}$ est l'ensemble des compacts héréditaires;

- $\mathcal{G}$ est l'ensemble des compacts héréditaires $K$ tels que $K \cap \mathbb{R}^{\omega}$ soit un fermé héréditaire grand;

- $\Psi$ est l'ensemble des compacts $K$ tels qu'il y ait $x \in X$ pour lequel on ait $\operatorname{Her}(K) \cap \mathbb{R} x=\{0\}$.

On appelle bonne suite de compacts de $\boldsymbol{K}$ toute suite $\left(K_{n}\right)_{n}$ telle que :

(a) pour tout $n, K_{n} \in \mathcal{G}$;

(b) $K_{0}=\boldsymbol{K}$ et la suite est décroissante : $K_{n} \supseteq K_{n+1}$ pour tout $n$;

(c) pour tout $n$, si on pose $F_{n}=K_{n} \cap \mathbb{R}^{\omega}$, on a $F_{n+1}+F_{n+1}+F_{n+1} \subseteq K_{n}$, c'est-à-dire que

$$
x, y, z \in K_{n+1} \cap \mathbb{R}^{\omega} \Rightarrow x+y+z \in K_{n} ;
$$

(d) pour tout compact $K \in \mathcal{G}$, il y a un $n$ tel que $K_{n} \subseteq K$;

(e) $\bigcap_{n} K_{n}=\{0\}$.

Le point fondamental est qu'il existe une telle suite. Pour le prouver, on va d'abord réduire le problème; en fait tout se ramène à construire une suite vérifiant (a) et (d).

Si une telle suite est construite, on peut aisément la rendre décroissante (on remplace un terme par l'intersection de ses prédécesseurs, qui reste dans $\mathcal{G}$ par (3.2.4)) et rajouter un premier terme égal à $\boldsymbol{K}$. On obtient (e) de toute 
suite vérifiant (d) puisqu'on peut facilement construire des fermés grands d'intersection réduite à 0 , et que l'intersection des termes de la suite est contenue dans cette intersection (par exemple : on prend des compacts dont les traces sur $\mathbb{R}^{\omega}$ sont les $\left.W_{N}\right)$.

Enfin (a) et (d) permettent de modifier la suite pour obtenir (c) : On part d'une suite $\left(J_{p}\right)_{p}$ vérifiant (d), (a) et aussi (b). Toute suite $K_{n}=J_{p_{n}}$ extraite avec $\left(p_{n}\right)_{n}$ strictement croissante vérifie toujours (a), (b) et (d).

Pour pouvoir extraire une suite vérifiant $(\mathrm{c})$, on se ramène à montrer que pour tout $K$ héréditaire à trace grande sur $\mathbb{R}^{\omega}$, il y a $K^{\prime}$ héréditaire à trace grande sur $\mathbb{R}^{\omega}$ tel que $K^{\prime} \vee K^{\prime} \subseteq K$ (on procède ainsi : si on a réussi à obtenir la propriété précédente et donc, après extraction, une suite $\left(M_{p}\right)_{p}$ vérifiant $M_{p+1} \vee M_{p+1} \subseteq M_{p}$, la suite $\left(N_{p}\right)_{p}=\left(2^{-p} M_{p}\right)_{p}$ vérifiera $\left(N_{p+1} \cap \mathbb{R}^{\omega}\right)+\left(N_{p+1} \cap \mathbb{R}^{\omega}\right) \subseteq N_{p}$ puisque si $x, y \in\left(N_{p+1} \cap \mathbb{R}^{\omega}\right)$, on a $2^{p}(x+y) \leq_{\omega} 2^{p}(2(x \vee y))=\left(2^{p+1} x\right) \vee\left(2^{p+1} x\right) \in M_{p}$; il suffit alors de considérer la suite $\left(N_{2 p}\right)_{p}$ pour avoir (a), (d) et (c)).

Soit $K \in \mathcal{G}$; posons pour $n>0$,

$$
A_{n}=\left\{(x, y) \in \boldsymbol{K}^{2} \mid n^{-1}(x \vee y) \in K\right\},
$$

qui est compact dans $\boldsymbol{K}^{2}$. Par hérédité de $K$ on a :

(i) $A_{n} \subseteq A_{n+1}$;

(ii) $\left(A_{n}\right)_{x}=\left\{y \mid(x, y) \in A_{n}\right\}$ est héréditaire pour tout $x \in \boldsymbol{K}$;

(iii) $\left(A_{n}\right)_{y} \subseteq\left(A_{n}\right)_{x}$ si $x \leq_{\omega} y$.

Soit $B_{n, p}=\left\{x \mid J_{p} \subseteq\left(A_{n}\right)_{x}\right\} \subseteq \boldsymbol{K}$. Par (iii) cet ensemble est héréditaire. De plus, $B_{n, p}$ est compact : en effet, il est fermé dans $\boldsymbol{K}$ car si $x \notin B_{n, p}$, on a $y \in J_{p}$ tel que $n^{-1}(x \vee y) \notin K$, et par continuité de $u \mapsto u \vee y$ on peut trouver un ouvert $O$ contenant $x$ et tel que $n^{-1}(O \vee y) \cap K=\emptyset$, d'où $O \cap B_{n, p}=\emptyset$.

Soit $x \in X$; pour tout $y \in X$, on a $x \vee y \in X$ et par (3.2.3) il y a un $n$ tel que $n^{-1}(x \vee y) \in K$, c'est-à-dire $(x, y) \in A_{n}$.

Ainsi $\bigcup_{n}\left(A_{n}\right)_{x} \supseteq X$, ce qui prouve que cet ensemble, qui est héréditaire par (ii), a une trace grande sur $\mathbb{R}^{\omega}$. Donc l'un des $\left(A_{n}\right)_{x} \cap \mathbb{R}^{\omega}$ a une trace grande sur $\mathbb{R}^{\omega}$ par (3.2.8), puisque la réunion de ces traces est la trace de la réunion. Donc les $J_{p}$ vérifiant $(\mathrm{d})$, il y a $p$ tel que $J_{p} \subseteq\left(A_{n}\right)_{x}$, c'est-à-dire $x \in B_{n, p}$.

On a donc $\bigcup_{n, p} B_{n, p} \supseteq X$; la trace de cette union de compacts héréditaires sur $\mathbb{R}^{\omega}$ contient $X$ et est donc grande; il résulte qu'elle a forcément un de ses termes grand. Il y a donc $n_{0}, p_{0}$ tels que $B_{n_{0}, p_{0}}$ ait sa trace grande sur $\mathbb{R}^{\omega}$. Posons $L=J_{p_{0}} \cap B_{n_{0}, p_{0}}$, compact à trace grande par (3.2.4). On a $L \times L \subseteq B_{n_{0}, p_{0}} \times J_{p_{0}} \subseteq A_{n_{0}}$ par définition des $A_{n}, B_{n, p}$, et si on pose $K^{\prime}=\left\{n_{0}^{-1} x \mid x \in L\right\}$, on a bien là un compact à trace grande sur $\mathbb{R}^{\omega}$, tel que $K^{\prime} \vee K^{\prime} \subseteq K$. 
Ainsi tout se ramène à trouver une suite vérifiant (a) et (d).

Or $\Psi$ est un $\sigma$-idéal de compacts grâce à (3.2.8), et cet idéal est analytique puisqu'on peut le définir par

$$
K \in \Psi \Leftrightarrow \exists x \in X, \forall n>0, n^{-1} x \notin \operatorname{Her}(K) .
$$

D'après le résultat de Kechris-Louveau-Woodin [K-L-W], il est en fait $\mathrm{G}_{\delta}$.

L'ensemble $\mathcal{G}$ est le complémentaire de $\Psi$, sur l'ensemble fermé des compacts héréditaires. $\mathcal{G}$ est donc un $\mathrm{F}_{\sigma}, \bigcup_{n} \mathcal{G}_{n}$, et si on réussit à trouver une suite vérifiant une $(\mathrm{d})$ restreinte pour chaque fermé $\mathcal{G}^{\prime}$ inclus dans $\mathcal{G}$, on aura en réunissant les suites correspondantes pour les $\mathcal{G}_{n}$ notre propriété (d) voulue.

Soit donc $\Phi \subseteq \mathcal{G}$ un fermé non vide de $\mathcal{K}(\boldsymbol{K})$.

FAIT. Il y a un ouvert relatif $U \subseteq \Phi$ tel que $\bigcap\{K \mid K \in U\} \in \mathcal{G}$.

Preuve. Considérons une base dénombrable d'ouverts relatifs de $\Phi$, notée $\left\{U_{n}\right\}_{n}$.

Posons $\Gamma=\bigcup_{n} \bigcap\left\{K \mid K \in U_{n}\right\}$. Alors $\Gamma$ rencontre $\mathbb{R}^{*} x$ pour tout $x \in X$. En effet, si $K \in \Phi$, par (3.2.3), on a $m>0$ tel que $m^{-1} x \in K$. Donc en posant

$$
\Phi_{m}=\left\{K \in \Phi \mid m^{-1} x \in K\right\},
$$

on voit que les $\Phi_{m}$ sont des fermés de $\Phi$ dont la réunion est $\Phi$. $\Phi$ est compact (fermé de $\mathcal{K}(\boldsymbol{K})$ ), donc de Baire, il y a donc un des $\Phi_{m}$ dont l'intérieur est non vide; par suite il y a $m, n$ tels que $U_{n} \subseteq \Phi_{m}$.

Cela veut dire que pour tout $K \in U_{n}$, on aura $m^{-1} x \in K$; d'où $m^{-1} x \in$ $\bigcap\left\{K \mid K \in U_{n}\right\} \subseteq \Gamma$. Donc $\Gamma \cap \mathbb{R}^{\omega}=\bigcup_{n} \bigcap\left(\left\{K \mid K \in U_{n}\right\} \cap \mathbb{R}^{\omega}\right)$ est un ensemble héréditaire grand, et par (3.2.8), $\left\{\left\{K \mid K \in U_{n}\right\} \cap \mathbb{R}^{\omega}\right.$ est grand pour un certain $n$.

Or $\bigcap\left\{K \mid K \in U_{n}\right\}$ est compact héréditaire, donc cela signifie bien qu'il appartient à $\mathcal{G}$, ce qui conclut la preuve du fait : $U=U_{n}$ convient.

Suite de la preuve de (3.2.10). On peut construire de proche en proche une suite transfinie $\Phi^{\alpha}$ de fermés inclus dans $\mathcal{G}$ comme suit :

- $\Phi^{0}=\Phi$

- si $\Phi^{\alpha} \neq \emptyset$, on applique le fait précédent à $\Phi^{\alpha}$ : il y a un ouvert relatif $U^{\alpha}$ de $\Phi^{\alpha}$ tel que $\bigcap\left\{K \mid K \in U^{\alpha}\right\}$ est dans $\mathcal{G}$; on pose $\Phi^{\alpha+1}=\Phi^{\alpha}-U^{\alpha}$;

- si $\alpha$ est limite, $\Phi^{\alpha}=\bigcap_{\lambda<\alpha} \Phi^{\lambda}$.

Une telle suite est forcément de longueur dénombrable, il y a donc un premier $\gamma<\omega_{1}$ tel que $\Phi^{\gamma}=\emptyset$. Prenons pour $K_{n}$ une énumération des ensembles $\bigcap\left\{K \mid K \in U^{\alpha}\right\}$ pour tous les $\alpha<\gamma$.

Comme $\Phi=\bigcup_{\alpha<\gamma} U^{\alpha}$, on a pour tout $L \in \Phi$ un $\alpha<\gamma$ tel que $L \in U^{\alpha}$, d'où un $n$ tel que $K_{n}=\bigcap\left\{K \mid K \in U^{\alpha}\right\} \subseteq L$. Donc les $K_{n}$ conviennent bien. 
Ainsi on a construit notre suite de compacts, par récurrence transfinie dans chaque $\mathcal{G}_{n}$.

On notera désormais $\left(K_{n}\right)_{n}$ notre bonne suite qui vérifie donc les conditions (a) à (e) spécifiées précédemment. La construction d'une évaluation à partir d'une telle suite à été traitée dans [C], on va ici en rappeler les grandes lignes.

On commence par définir des fonctions sur $c_{00}$. Pour tout $x \in c_{00}$, on pose

$$
\begin{aligned}
& \Psi_{0}(x)=\inf \left\{2^{-n} \mid x \in K_{n}\right\} \\
& \Psi_{1}(x)=\inf \left\{\sum_{1 \leq k \leq p} \Psi_{0}\left(x_{k}\right) \mid p \geq 1, x_{k} \in c_{00}, x \leq_{\omega} \sum_{1 \leq k \leq p} x_{k}\right\} .
\end{aligned}
$$

FAIT. Pour tous $x, y \in c_{00}$, on $a$ :

- $0 \leq \frac{1}{2} \Psi_{0}(x) \leq \Psi_{1}(x) \leq \Psi_{0}(x) \leq 1$ et $\Psi_{0}(x)=0 \Leftrightarrow x=0$.

- $\Psi_{1}(x+y) \leq \Psi_{1}(x)+\Psi_{1}(y)$ et $x \leq_{\omega} y \Rightarrow \Psi_{1}(x) \leq \Psi_{1}(y)$.

- Pour tout $n, \Psi_{1}$ est continue sur $\mathbb{R}^{n}$.

Preuve. La seule propriété un peu délicate est $\Psi_{0}(x) \leq 2 \Psi_{1}(x)$, ou encore

$$
x \leq_{\omega} \sum_{1 \leq k \leq p} x_{k} \Rightarrow \Psi_{0}(x) \leq 2 \sum_{1 \leq k \leq p} \Psi_{0}\left(x_{k}\right) .
$$

On se ramène facilement à prouver que pour $p \geq 1$, et $x_{1}, \ldots, x_{p} \in c_{00}$, on a

$$
\Psi_{0}\left(\sum_{1 \leq k \leq p} x_{k}\right) \leq 2 \sum_{1 \leq k \leq p} \Psi_{0}\left(x_{k}\right)
$$

On montre ceci par récurrence sur $p$. Si $p=1$, cela s'écrit $\Psi_{0}\left(x_{1}\right) \leq 2 \Psi_{0}\left(x_{1}\right)$ et il n'y a rien à prouver. Supposons que c'est vrai pour toute famille de $p$ suites finies, et montrons-le pour $x_{1}, \ldots, x_{p+1} \in c_{00}$.

Quitte à changer l'ordre on peut supposer que $\Psi_{0}\left(x_{1}\right) \leq \Psi_{0}\left(x_{k}\right)$ pour tout $k$, et que les $x_{k}$ sont non nulles, sans quoi on est tout de suite ramené au cas de $p$ suites. On a donc

$$
\Psi_{0}\left(x_{1}\right) \leq \frac{1}{2} \sum_{1 \leq k \leq p+1} \Psi_{0}\left(x_{k}\right)
$$

et il y a un premier $r \leq 1$ tel que

$$
\sum_{1 \leq k \leq r+1} \Psi_{0}\left(x_{k}\right)>\frac{1}{2} \sum_{1 \leq k \leq p+1} \Psi_{0}\left(x_{k}\right) .
$$

On aura donc par hypothèse de récurrence,

$$
\Psi_{0}\left(\sum_{1 \leq k \leq r} x_{k}\right) \leq 2 \sum_{1 \leq k \leq r} \Psi_{0}\left(x_{k}\right) \leq \sum_{1 \leq k \leq p+1} \Psi_{0}\left(x_{k}\right),
$$




$$
\begin{aligned}
& \Psi_{0}\left(\sum_{r+1<k \leq p+1} x_{k}\right) \leq 2 \sum_{r+1<k \leq p+1} \Psi_{0}\left(x_{k}\right) \leq \sum_{1 \leq k \leq p+1} \Psi_{0}\left(x_{k}\right), \\
& \Psi_{0}\left(x_{r+1}\right) \leq \sum_{1 \leq k \leq p+1} \Psi_{0}\left(x_{k}\right) .
\end{aligned}
$$

Comme les $K_{n}$ forment une bonne suite de compacts, on a $x, y, z \in K_{n} \Rightarrow$ $x+y+z \in K_{n-1}$, d'où $\Psi_{0}(x), \Psi_{0}(y), \Psi_{0}(z) \leq r \Rightarrow \Psi_{0}(x+y+z) \leq 2 r$.

Donc on a bien

$$
\Psi_{0}\left(\sum_{1 \leq k \leq p+1} x_{k}\right) \leq 2 \sum_{1 \leq k \leq p+1} \Psi_{0}\left(x_{k}\right),
$$

et la récurrence est achevée.

Fin de la preuve de (3.2.10). On définit ensuite la fonction $\varphi_{0}$ :

$$
\forall x \in \mathbb{R}^{\omega}, \quad \varphi_{0}(x)=\sup \left\{\Psi_{1}\left(\pi_{n}(x)\right) \mid n \in \omega\right\},
$$

et à partir d'elle notre évaluation

$$
\varphi(x)=\sup \left\{r^{-1} \varphi_{0}(r x) \mid r \geq 1\right\} .
$$

Comme dans $[\mathrm{C}]$, on montre que c'est une évaluation et que $\varphi_{0} \leq \varphi \leq 2 \varphi_{0}$.

La seule différence avec la construction de [C] est qu'ici on a

$$
\operatorname{Exh}(\varphi) \subseteq X \subseteq \operatorname{Fin}(\varphi)
$$

Vérifions-le : Si $x \in X$, pour tout $n, K_{n}$ est à trace grande, donc il y a un $\lambda>0$ tel que $\lambda x \in K_{n}$ et donc pour tout $m, \pi_{m}(\lambda x) \in K_{n}$ et finalement $\Psi_{0}\left(\pi_{m}(\lambda x)\right) \leq 2^{-n}$. Ceci entraîne $\Psi_{1}\left(\pi_{m}(\lambda x)\right) \leq 2^{-n}$ pour tout $m$, et donc $\varphi(\lambda x) \leq 2 \varphi_{0}(\lambda x) \leq 2^{-n+1}$. Finalement la monotonie de $\varphi$ permet de conclure :

$$
\lim _{\lambda \rightarrow 0} \varphi(\lambda x)=0 .
$$

Montrons $x \notin X \Rightarrow x \notin \operatorname{Exh}(\varphi)$. Soit $x \notin X$. Il suffit d'exhiber un compact $K$ à trace grande qui ne contient aucun élément du type $m^{-1} \pi_{m}(x)+$ $\varepsilon_{m}(x)$. En prenant un $K_{n_{0}}$ inclus dans ce compact, il ne contient aucun de ces éléments non plus. On a donc

$$
\begin{aligned}
\forall m, & m^{-1} \pi_{m}(x)+\varepsilon_{m}(x) \notin K_{n_{0}} \Rightarrow \forall q, \forall m>q, m^{-1} \pi_{q}(x)+\varepsilon_{q}(x) \notin K_{n_{0}} \\
\Rightarrow & \forall q, \quad \forall m^{\prime}>m>q, \quad \Psi_{0}\left(\pi_{m^{\prime}}\left(m^{-1} \pi_{q}(x)+\varepsilon_{q}(x)\right)\right) \geq 2^{-n_{0}} \\
\Rightarrow & \forall q, \quad \forall m^{\prime}>m>q, \\
& \Psi_{1}\left(\pi_{m^{\prime}}\left(m^{-1} \pi_{q}(x)+\varepsilon_{q}(x)\right)\right) \geq \Psi_{0}\left(\pi_{m^{\prime}}\left(m^{-1} \pi_{q}(x)+\varepsilon_{q}(x)\right)\right) / 2 \geq 2^{1-n_{0}} \\
\Rightarrow & \forall q, \quad \forall m>q, \quad \varphi\left(m^{-1} \pi_{q}(x)+\varepsilon_{q}(x)\right) \geq \varphi_{0}\left(m^{-1} \pi_{q}(x)+\varepsilon_{q}(x)\right) \geq 2^{1-n_{0}}
\end{aligned}
$$


ce qui entraîne, d'après l'inégalité triangulaire et la continuité de $\varphi \operatorname{sur} \mathbb{R}^{q}$, que $\varphi\left(\varepsilon_{q}(x)\right) \geq 2^{1-n_{0}}$ pour tout $q$ et finalement $x \notin \operatorname{Exh}(\varphi)$. On a donc bien $\operatorname{Exh}(\varphi) \subseteq X$.

Tout revient donc à exhiber $K$. L'ensemble $\mathcal{I}$ des parties $A$ de $\omega$ telles que $\pi_{A}(x) \in X$ est un idéal analytique, donc par le théorème de Talagrand [T] on peut partitionner $\omega=\bigcup_{n} O_{n}$ avec $O_{n}$ fini et une union de $O_{n}$ est dans $\mathcal{I}$ si et seulement si c'est une union finie. Quitte à répartir les éléments des $O_{n}$ tels que $\pi_{O_{n}}(x)=0$ sur les autres $O_{n^{\prime}}$, on peut supposer que $\pi_{O_{n}}(x) \neq 0$ pour tout $n$.

Considérons le compact $K$ des suites $y \in \boldsymbol{K}$ telles que pour tout $n$ il y ait $k \in O_{n}$ tel que $x(k) \neq 0$ et $|y(k)| \leq \frac{1}{2}|x(k)|$.

$K$ est clairement héréditaire et il est grand : si $y \in X$, il n'y a qu'un nombre fini de $n$ tels que $\frac{1}{2} \pi_{O_{n}}(x) \leq_{\omega} \pi_{O_{n}}(y)$, puisque pour la réunion $U$ de ces $O_{n}$ on a $\pi_{U}(x) \leq_{\omega} 2 \pi_{U}(y) \in X$ et $U \in \mathcal{I}$. Il y a donc $n_{0}$ à partir duquel on a pour chaque $n$ un $k_{n} \in O_{n}$ tel que $\left|y\left(k_{n}\right)\right|<\frac{1}{2}\left|x\left(k_{n}\right)\right|$. Dans chaque $O_{n}$ avec $n<n_{0}$, on a un $k_{n}$ tel que $x\left(k_{n}\right) \neq 0$. Donc si on prend un $\lambda<1$ assez petit, on aura pour $n<n_{0}$,

$$
\left|\lambda y\left(k_{n}\right)\right|<\frac{1}{2}\left|x\left(k_{n}\right)\right|,
$$

et pour $n \geq n_{0}$,

$$
\left|\lambda y\left(k_{n}\right)\right| \leq\left|y\left(k_{n}\right)\right|<\frac{1}{2}\left|x\left(k_{n}\right)\right| .
$$

Ainsi $\lambda y \in K$ et $K \in \mathcal{G}$.

Si on avait $y=m^{-1} \pi_{m}(x)+\varepsilon_{m}(x) \in K$, en prenant $n_{0}$ assez grand pour que $\{0,1, \ldots, m\} \subseteq O_{1} \cup \ldots \cup O_{n_{0}-1}$, on aurait pour $n \geq n_{0}$ certainement aucun $k \in O_{n}$ tel que $x(k) \neq 0$ et $|y(k)| \leq \frac{1}{2}|x(k)|$, car cela s'écrit $0<$ $|x(k)| \leq \frac{1}{2}|x(k)|$, ce qui est absurde. Donc $y \notin K$, et $K$ convient. Du même coup l'encadrement de $X$ est démontré.

Pour conclure la preuve de (3.2.10), on remarque que les exigences d'unicité sont assurées par (2.3.7).

Du coup, le théorème (3.2.1) est entièrement démontré.

On a donc obtenu un encadrement canonique de nos espaces héréditaires, que l'on avait déjà en (2.3.7) pour les espaces complètement métrisables. Les questions naturelles qui viennent alors sont de savoir si on peut avoir un tel encadrement et plonger $c_{00}$ quand même, et si on peut trouver des espaces dans lesquels $c_{00}$ ne se plonge pas du tout mais qui ne sont pas "évidents" : par exemple qui soient complètement métrisables mais pas fermés dans leur $\operatorname{Fin}(\varphi)$ correspondant.

On va voir que la réponse est positive dans les deux cas. Néanmoins, on va voir d'abord que (3.2.10) permet de connaître complètement les espaces $E$ qui sont très simples du point de vue descriptif : 
Proposition (3.2.11). Si $X$ est un espace héréditaire qui est un $\boldsymbol{\Sigma}_{3}^{0}$ de $\mathbb{R}^{\omega}$, alors on a la trichotomie :

- $c_{00} \leq_{\operatorname{lin}}^{+} X$;

- $X=\operatorname{Fin}(\varphi)$ pour une évaluation et $X$ est en fait un $\Sigma_{2}^{0}$, avec $\ell_{\infty} \leq X$;

- $X=\operatorname{Exh}(\varphi)=\operatorname{Fin}(\varphi)$ pour une évaluation et $X$ est en fait un $\boldsymbol{\Sigma}_{2}^{0}$.

Preuve. Si on n'a pas $c_{00} \leq_{\operatorname{lin}}^{+} X$, on peut d'après (3.2.1) trouver $\varphi$ telle que $\operatorname{Exh}(\varphi) \subseteq X \subseteq \operatorname{Fin}(\varphi)$. Or un résultat de $[\mathrm{C}]$ (proposition (4.2.1)) est que si un espace obéit à un tel encadrement et est distinct de $\operatorname{Fin}(\varphi)$, il est $\boldsymbol{\Pi}_{3}^{0}$-hard (cf. $[\mathrm{K}]$ ), donc ne peut pas être $\boldsymbol{\Sigma}_{3}^{0}$; on a donc nécessairement $X=\operatorname{Fin}(\varphi)$ et le reste de la dichotomie en découle compte tenu des résultats de $[\mathrm{C}]$.

\subsection{Quelques situations possibles}

Proposition (3.3.1). On peut trouver un espace héréditaire $X$ analytique dans $\mathbb{R}^{\omega}$ tel que

$$
c_{0} \subseteq X \subseteq \ell_{\infty}, \quad c_{00} \leq_{\operatorname{lin}}^{+} X
$$

Preuve. Considérons une partition $\omega=\bigcup_{n} A_{n}$ de $\omega$ en parties infinies. Pour $I=(1,1, \ldots)$ et pour chaque $n$, posons $a_{n}=\pi_{A_{n}}(I)$, qui est en fait la fonction caractéristique de $A_{n}$. On pose

$$
X=\left\{x \mid \exists t \in c_{00}, \exists h \in c_{0}, \quad x \leq_{\omega} \sum_{n} t(n) a_{n}+h\right\} .
$$

Alors $X$ est clairement un espace héréditaire analytique, et vérifie la première condition. Par ailleurs, $t \mapsto \sum_{n} t(n) a_{n}$ est le plongement voulu puisque les $A_{n}$ étant infinis, un $a_{n}$ n'est jamais dominé par un élément de $c_{0}$, donc une somme $\sum_{n} t(n) a_{n}$ avec $t \notin c_{00}$ n'est jamais dominée par une somme de la forme $\sum_{n} s(n) a_{n}+h^{\prime}$ avec $s \in c_{00}, h^{\prime} \in c_{0}$.

Pour rendre plus commode l'énoncé suivant, on introduit la définition suivante :

DÉfinition (3.3.2). Une suite $\left(x_{n}\right)_{n}$ d'éléments de $\mathbb{R}^{\omega}$ est dite totalement additive si pour chaque $t \in \mathbb{R}^{\omega}$, la série $t(0) x_{0}+t(1) x_{1}+t(2) x_{2}+\ldots$ converge dans $\mathbb{R}^{\omega}$ pour la topologie produit.

Proposition (3.3.3). (i) Une suite $\left(x_{n}\right)_{n}$ est totalement additive si et seulement si pour tout $k$, la famille de réels $\left(x_{n}(k)\right)_{n}$ est à support fini.

(ii) Si les $\left(x_{n}\right)_{n}$ ont des supports deux à deux disjoints, $\left(x_{n}\right)_{n}$ est totalement additive.

Preuve. En effet, d'après (3.3.2) la totale additivité signifie que pour tout $k$, et tout $t \in \mathbb{R}^{\omega}$, la série $t(0) x_{0}(k)+t(1) x_{1}(k)+t(2) x_{2}(k)+\ldots$ est 
convergente dans $\mathbb{R}$, ce qui est vérifié seulement si $\left(x_{i}(k)\right)_{k}$ est à support fini. La deuxième assertion est une conséquence évidente de la première.

Proposition (3.3.4). Soient $\varphi$ et $\psi$ deux évaluations et $\left(x_{n}\right)_{n}, x_{n} \in$ $\left(\mathbb{R}^{+}\right)^{\omega}$, vérifiant les conditions suivantes :

(a) pour tout $n, x_{n} \in \operatorname{Fin}(\varphi)-\operatorname{Exh}(\varphi)$ et $\sum_{n} x_{n}$ converge dans $\operatorname{Fin}(\varphi)$ muni de $\varphi$, vers $s \in \operatorname{Fin}(\varphi)$;

(b) les $\left(x_{n}\right)_{n}$ ont des supports disjoints;

(c) $I=(1,1, \ldots) \notin \operatorname{Fin}(\psi)$.

Alors il existe un couple $(X, d)$ tel que:

(i) $X$ est un espace héréditaire analytique, et $d$ une distance complète monotone sur $X$, compatible avec la structure vectorielle de $X$;

(ii) $\operatorname{Exh}(\varphi) \subseteq X \subseteq \operatorname{Fin}(\varphi)$;

(iii) $X$ n'est pas fermé dans $\operatorname{Fin}(\varphi): x_{n} \in X$ pour chaque $n$ mais $s \notin X$;

(iv) $\operatorname{Fin}(\psi) \leq_{\operatorname{lin}}^{+} X$.

Preuve. On définit $X$ comme l'ensemble des $y \in \operatorname{Fin}(\varphi)$ vérifiant

$$
\exists t \in \operatorname{Fin}(\psi), \exists h \in \operatorname{Exh}(\varphi), \quad y \leq_{\omega} \sum_{n} t(n) x_{n}+h,
$$

et si $y \in X$, on pose

$$
\begin{aligned}
& \alpha(y)=\inf \left\{\psi(t) \mid \exists h \in \operatorname{Exh}(\varphi), y \leq_{\omega} \sum_{n} t(n) x_{n}+h\right\}, \\
& \delta(y)=\alpha(y)+\varphi(y) .
\end{aligned}
$$

Ces définitions entraînent :

(1) $\quad X$ est un sous-espace vectoriel analytique de $\mathbb{R}^{\omega}$.

Par exemple, $y \leq_{\omega} \sum_{n} t(n) x_{n}+h$ et $y^{\prime} \leq_{\omega} \sum_{n} t^{\prime}(n) x_{n}+h^{\prime}$ entraînent, comme les $x_{n}$ sont à composantes positives, que

$$
y+y^{\prime} \leq_{\omega} \sum_{n}\left(|t|+\left|t^{\prime}\right|\right)(n) x_{n}+|h|+\left|h^{\prime}\right|
$$

avec $|t|+\left|t^{\prime}\right| \in \operatorname{Fin}(\psi)$ et $|h|+\left|h^{\prime}\right| \in \operatorname{Exh}(\varphi)$; quant à l'analycité, elle provient de la forme de la définition $\exists y, \exists h, \ldots$, et du fait que $\operatorname{Fin}(\psi), \operatorname{Exh}(\varphi)$ et enfin $\leq_{\omega}$ dans $\mathbb{R}^{\omega} \times \mathbb{R}^{\omega}$ sont des boréliens.

(2) $\operatorname{Exh}(\varphi) \subseteq Y \subseteq \operatorname{Fin}(\varphi)$.

C'est évident sur la définition.

$$
\begin{aligned}
& \alpha\left(y+y^{\prime}\right) \leq \alpha(y)+\alpha\left(y^{\prime}\right) \\
& y \leq \omega y^{\prime} \in X \Rightarrow \alpha(y) \leq \alpha\left(y^{\prime}\right), \\
& \alpha(\lambda y) \leq \sup (1,|\lambda|) \alpha(y) \\
& y \in \operatorname{Exh}(\varphi) \Rightarrow \alpha(y)=0 .
\end{aligned}
$$


Ces propriétés découlent directement des propriétés d'évaluations de $\psi$, et de manipulations sur les $\leq_{\omega}$ que l'on prend soin d'opérer sur des suites à composantes positives.

$$
\text { Si } y \in X \text {, on } a \lim _{\lambda \rightarrow 0} \alpha(\lambda y)=0 \text {. }
$$

En effet, considérons $t \in \operatorname{Fin}(\psi)$ et $h \in \operatorname{Exh}(\varphi)$, tous deux à composantes positives, tels que $y \leq_{\omega} \sum_{n} t(n) x_{n}+h$. Alors on a aussi

$$
\lambda y \leq_{\omega} \sum_{n}(\lambda t)(n) x_{n}+\lambda h
$$

et donc $\alpha(\lambda y) \leq \psi(\lambda t) \rightarrow 0$.

(5) Si les $y_{m} \in X$, à composantes positives, vérifient $\delta\left(y_{m}\right) \leq 2^{-m}$, alors on a un unique $z \in X$ tel que $\delta\left(z-\sum_{k \leq m} y_{k}\right) \rightarrow 0$ pour $m \rightarrow \infty$, et $z$ est la somme des $y_{m}$, calculée pour la topologie complète $\operatorname{Fin}(\varphi)$ ou pour celle de $\mathbb{R}^{\omega}$.

Les hypothèse impliquent à la fois $\alpha\left(y_{m}\right) \leq 2^{-m}$ et $\varphi\left(y_{m}\right) \leq 2^{-m}$. Donc, $\operatorname{Fin}(\varphi)$ étant complet, la famille $\left(y_{m}\right)_{m}$ est sommable dans $\operatorname{Fin}(\varphi) ; z$ sera sa somme. Par définition de $\alpha$, on a pour chaque $m$ un $t_{m} \in \operatorname{Fin}(\psi)$ et un $h_{m} \in \operatorname{Exh}(\varphi)$, qu'on peut tous choisir à composantes positives, tels que

$$
y_{m} \leq_{\omega} \sum_{n} t_{m}(n) x_{n}+h_{m} \quad \text { et } \quad \psi\left(t_{m}\right) \leq 2^{1-m} .
$$

Notons tout d'abord que, dans Fin $(\psi)$, la famille $t_{m}$ est sommable, et on peut appeler $t$ sa somme. De plus pour chaque composante $k$, on a

$$
0 \leq y_{m}(k) \leq \sum_{n} t_{m}(n) x_{n}(k)+h_{m}(k),
$$

et donc aussi

$$
0 \leq y_{m}(k) \leq \sum_{n} t_{m}(n) x_{n}(k)+\inf \left(h_{m}(k), y_{m}(k)\right),
$$

c'est-à-dire qu'on peut remplacer chaque $h_{m}$ par $h_{m} \wedge y_{m}$, qui reste dans $X$ par hérédité, et finalement on voit qu'on peut choisir les $h_{m}$ tels que $\varphi\left(h_{m}\right) \leq \varphi\left(y_{m}\right) \leq 2^{-m}$.

La famille $h_{m}$ est sommable dans $\operatorname{Exh}(\varphi)$, et en additionnant les inégalités $(*)$, ce qui est possible, toutes les composantes étant positives, il vient, en effectuant le calcul dans $\mathbb{R}^{\omega}$,

$$
\begin{aligned}
\sum_{m_{0} \leq m} y_{m} & \leq{ }_{\omega} \lim _{m \rightarrow \infty} \sum_{m_{0} \leq p \leq m}\left(\sum_{n} t_{p}(n) x_{n}+h_{p}\right) \\
& =\lim _{m \rightarrow \infty}\left(\sum_{n}\left(\sum_{m_{0} \leq p \leq m} t_{p}(n)\right) x_{n}+\sum_{m_{0} \leq p \leq m} h_{p}\right)
\end{aligned}
$$




$$
=\sum_{n}\left(\sum_{m_{0} \leq m} t_{m}\right)(n) x_{n}+\sum_{m_{0} \leq m} h_{m} .
$$

Ceci entraîne deux choses ; d'abord, pour $m_{0}=0$, on obtient $z \leq_{\omega} \sum_{n} t(n) x_{n}$ $+h$ avec $t \in \operatorname{Fin}(\psi)$ et $h \in \operatorname{Exh}(\varphi)$, donc $z \in X$.

Ensuite la formule pour chaque $m_{0}$ donne

$$
z-\sum_{m<m_{0}} y_{m}=\sum_{m_{0} \leq m} y_{m} \leq_{\omega} \sum_{n}\left(\sum_{m_{0} \leq m} t_{m}\right)(n) x_{n}+\sum_{m_{0} \leq m} h_{m}
$$

avec $\sum_{m_{0} \leq m} h_{m} \in \operatorname{Exh}(\varphi)$ et $\sum_{m_{0} \leq m} t_{m} \in \operatorname{Fin}(\psi)$, et même

$$
\psi\left(\sum_{m_{0} \leq m} t_{m}\right) \leq \sum_{m_{0} \leq m} \psi\left(t_{m}\right) \leq \sum_{m_{0} \leq m} 2^{1-m} \leq 2^{2-m_{0}} \rightarrow 0
$$

quand $m_{0} \rightarrow \infty$. L'assertion est donc démontrée, puisqu'on a à la fois

$$
\alpha\left(z-\sum_{m<m_{0}} y_{m}\right) \leq 2^{2-m_{0}} \rightarrow 0 \quad \text { et } \varphi\left(z-\sum_{m<m_{0}} y_{m}\right) \rightarrow 0 .
$$

(6) $d\left(y, y^{\prime}\right)=\delta\left(y-y^{\prime}\right)$ définit sur $X$ une distance complète compatible avec la structure d'espace vectoriel héréditaire, qui coïncide sur $\operatorname{Exh}(\varphi)$ avec celle induite par $\varphi$.

D'après (3) et l'inégalité $\delta \geq \varphi$ qui assure que $d\left(y, y^{\prime}\right)=0 \Rightarrow y=y^{\prime}$, $d$ est une distance compatible avec la structure de groupe additif de $X$; la monotonie dans (3), et (4) assurent la compatibilité avec la structure vectorielle. (3) prouve aussi que $\delta=\varphi \operatorname{sur} \operatorname{Exh}(\varphi)$. Reste à montrer la complétude. Si $\left(y_{m}\right)_{m}$ est $d$-Cauchy, elle est $\varphi$-Cauchy et a une limite $z \in$ $\operatorname{Fin}(\varphi)$. Il suffit de montrer qu'une sous-suite de $\left(y_{m}\right)_{m}$ est $d$-convergente vers $z$. Or on peut extraire une sous-suite $y_{k}^{\prime}=y_{m_{k}}$ telle que $d\left(y_{k}^{\prime}, y_{k+1}^{\prime}\right) \leq 2^{-k}$.

Alors $\delta\left(y_{k}^{\prime}-y_{k+1}^{\prime}\right) \leq 2^{-k}$, donc d'après (5) la suite $\left(\left|y_{k}^{\prime}-y_{k+1}^{\prime}\right|\right)_{k}$ est $d$-convergente vers un $z^{\prime} \in X$ qui est forcément égal à $\sum_{k}\left|y_{k}^{\prime}-y_{k+1}^{\prime}\right|$ calculé dans $\mathbb{R}^{\omega}$, de sorte qu'on a

$$
z-y_{k}^{\prime}=\sum_{l \geq k} y_{l+1}^{\prime}-y_{l}^{\prime} \leq_{\omega} \sum_{l \geq k}\left|y_{l}^{\prime}-y_{l+1}^{\prime}\right| \leq_{\omega} z^{\prime},
$$

ce qui prouve à la fois que $z \in X$ et que, grâce à la monotonie de $\delta$, $\lim _{k \rightarrow \infty} d\left(z, y_{k}^{\prime}\right)=0$.

$s \notin X$.

Raisonnons par l'absurde : supposons qu'il y a $t \in \operatorname{Fin}(\psi)$ et $h \in \operatorname{Exh}(\varphi)$ tels que $s \leq_{\omega} \sum_{n} t(n) x_{n}+h$. Ceci se lit, sur la composante $k$, qui est au plus dans le support de $x_{n}$ pour un seul $n=n_{0}$ d'après (b), compte tenu de la définition de $s$,

$$
s(k)=x_{n_{0}}(k) \leq t\left(n_{0}\right) x_{n_{0}}(k)+h(k) .
$$


On a sûrement une infinité de $k$ dans le support de $x_{n_{0}}$ tels que $h(k)<$ $\frac{1}{2} x_{n_{0}}(k)$, sans quoi on aurait $\frac{1}{2} x_{n_{0}} \leq_{\omega} h$ modulo une suite finie, d'où $x_{n_{0}} \in$ $\operatorname{Exh}(\varphi)$, ce qui contredirait (a). Il y a donc au moins un $k$ pour lequel (**) donne

$$
0<x_{n_{0}}(k) \leq t\left(n_{0}\right) x_{n_{0}}(k)+h(k)<t\left(n_{0}\right) x_{n_{0}}(k)+\frac{1}{2} x_{n_{0}}(k)
$$

et donc $0<\frac{1}{2} x_{n_{0}}(k)<t\left(n_{0}\right) x_{n_{0}}(k)$, et enfin $\frac{1}{2}<t\left(n_{0}\right)$.

Or ceci est vrai pour chaque $n_{0}$, donc on obtient

$$
\frac{1}{2} I=\left(\frac{1}{2}, \frac{1}{2}, \frac{1}{2}, \ldots\right) \leq_{\omega} t \in \operatorname{Fin}(\psi),
$$

ce qui contredit (c).

(8) $\operatorname{Fin}(\psi) \leq \leq_{\operatorname{lin}}^{+} X$.

Le même raisonnement qu'au (7) prouve que si

$$
\sum_{n} u(n) x_{n} \leq \omega \sum_{n} t(n) x_{n}+h
$$

avec $u, t \in \operatorname{Fin}(\psi)$, et $h \in \operatorname{Exh}(\varphi)$, alors pour chaque $n$ tel que $u(n) \neq 0$, on aura $\frac{1}{2}|u(n)| \leq|t(n)|$. Si $u(n)=0$, cela reste vrai évidemment. Ainsi

$$
\sum_{n} u(n) x_{n} \leq_{\omega} \sum_{n} t(n) x_{n}+h \Rightarrow u \leq_{\omega} 2 t .
$$

La fonction $f: t \mapsto \sum_{n} t(n) x_{n}$ est définie sur $\mathbb{R}^{\omega}$ puisque $\left(x_{n}\right)_{n}$ est totalement additive, elle est continue (pour la topologie de $\mathbb{R}^{\omega}$ à l'arrivée comme au départ), elle est monotone, vérifie par définition de $X$ que $f(\operatorname{Fin}(\psi)) \subseteq X$, et ce qui précède s'écrit $f(u) \in X \Rightarrow u \in \operatorname{Fin}(\psi)$. Donc $f$ est le plongement voulu.

EXEMPle (3.3.5). Considérons une partition de $\omega$ en une suite de parties infinies, $\omega=\bigcup_{n} A_{n}$, et posons $x_{n}=n^{-1} \pi_{A_{n}}(I)$. Considérons l'ensemble $X$ des $y \in \ell_{\infty}$ qui vérifient : il y a $t \in \ell_{1}$ et $h \in c_{0}$ tels que $y \leq_{\omega}$ $\sum_{n} t(n) x_{n}+h$. Alors $X$ est un sous-espace héréditaire analytique de $\mathbb{R}^{\omega}$ qui vérifie $c_{0} \subseteq X \subseteq \ell_{\infty}$, non fermé pour le norme de $\ell_{\infty}$, mais qui est complètement métrisable et même est un Banach. Une norme possible est donnée par

$$
n(y)=\inf \left\{\|t\|_{1} \mid t \in \ell_{1} \text { et } \exists h \in c_{0}, \quad y \leq_{\omega} \sum_{n} t(n) x_{n}+h\right\}+\|y\|_{\infty} .
$$

Preuve. C'est un simple cas particulier du (7) ; le fait qu'on obtient une norme, c'est-à-dire une évaluation homogène, est évident sur la définition.

On va maintenant construire un espace héréditaire non encadrable par un couple $(\operatorname{Exh}(\varphi), \operatorname{Fin}(\varphi))$, mais dans lequel $c_{00}$ ne se plonge pas. Cela prouvera que des hypothèses sur la complexité descriptive des espaces pour lesquels les conclusions de (3.2.1) s'appliqueraient sont nécessaires. En 
(3.2.1) on suppose $X$ analytique; le contre-exemple construit ici est au moins $\boldsymbol{\Sigma}_{2}^{1}$, si on rajoute un axiome adéquat qui fournit un p-point (sur les p-points cf. [J], ch. $4, \S 24$, p. 257 ), qui sera au moins $\boldsymbol{\Delta}_{2}^{1}$, mais on n'entrera pas dans ces considérations ici.

Proposition (3.3.6). (i) $S^{\prime} i l$ existe un ultrafiltre non principal $\mathcal{U}$ sur $\omega$, il y a un espace héréditaire $X \neq \mathbb{R}^{\omega}$ qui n'est contenu dans aucun $\operatorname{Fin}(\varphi) \neq \mathbb{R}^{\omega}$.

(ii) Si $\mathcal{U}$ est un p-point, on peut de plus exiger $c_{00} \not_{\operatorname{lin}} X$.

(iii) $(Z F C)+(M A)$ ou $(Z F C)+(C H)$ ou $(Z F+V=L)$. Il y a un $X$ héréditaire tel que $c_{00} \mathbb{L}_{\operatorname{lin}} X$ mais que $X$ ne soit pas complètement métrisable, et qu'on n'ait aucune $\varphi$ telle que $\operatorname{Exh}(\varphi) \subseteq X \subseteq \operatorname{Fin}(\varphi)$.

Preuve. (i) On pose $X=\left\{x \mid \exists A \in \mathcal{U}, \pi_{A}(x) \in \ell_{\infty}\right\}$. Alors $X$ est clairement un espace héréditaire, distinct de $\mathbb{R}^{\omega}$ car, par exemple, $(1,2,3, \ldots) \notin$ $X$. Mais si l'évaluation $\varphi$ vérifie $\operatorname{Fin}(\varphi) \neq \mathbb{R}^{\omega}$, considérons $x \notin \operatorname{Fin}(\varphi)$. Alors les $B \subseteq \omega$ tels que $\pi_{B}(x) \in \operatorname{Fin}(\varphi)$ forment un idéal borélien $\mathcal{J}$ pour lequel on peut décomposer $\omega=\bigcup_{n} F_{n}$ suivant la méthode de Talagrand. Les ensembles $F_{1} \cup F_{3} \cup F_{5} \cup \ldots$ et $F_{0} \cup F_{2} \cup F_{4} \cup \ldots$ ne sont donc pas dans cet idéal, et sont complémentaires l'un de l'autre; donc l'un des deux, appelonsle $A$, est dans $\mathcal{U}$, puisque c'est un ultrafiltre. Alors si $y=\pi_{A}(I)+\varepsilon_{A}(x)$, on a $y \in X$ car $\pi_{A}(y)=\pi_{A}(1) \in \ell_{\infty}$ avec $A \in \mathcal{U}$, et $y \notin \operatorname{Fin}(\varphi)$ car $\pi_{\omega-A}(y)=\pi_{\omega-A}(x) \notin \operatorname{Fin}(\varphi)$ puisque $\omega-A \notin \mathcal{J}$. Ainsi $X \nsubseteq \operatorname{Fin}(\varphi)$. Ceci est vrai pour toute évaluation telle que $\operatorname{Fin}(\varphi) \neq \mathbb{R}^{\omega}$, et on n'aura donc pas $\operatorname{Exh}(\varphi) \subseteq X \subseteq \operatorname{Fin}(\varphi)$. Mais si $\operatorname{Fin}(\varphi)=\mathbb{R}^{\omega}$, on n'a pas non plus l'encadrement car alors $\operatorname{Exh}(\varphi)=\operatorname{Fin}(\varphi)=\mathbb{R}^{\omega}$.

(ii) $\mathrm{Si}$ de plus $\mathcal{U}$ est un p-point, soit $x_{0}, x_{1}, x_{2}, \ldots \in X$ quelconques. On a $A_{0}, A_{1}, A_{2}, \ldots \in \mathcal{U}$ tels que $\pi_{A_{k}}\left(x_{k}\right) \in \ell_{\infty}$. On peut supposer les $A_{k}$ décroissants, quitte à en prendre des intersections finies, ce qui ne change rien, ces intersections restant dans $\mathcal{U}$. Comme $\mathcal{U}$ est un p-point il y a un $A \in \mathcal{U}$ tel que $A-A_{k}$ soit fini pour tout $k$. Alors on a aussi $\pi_{A}\left(x_{k}\right) \in \ell_{\infty}$ pour tout $k$.

Maintenant construisons une suite $(e(n))_{n}$ comme suit :

- $e(0)>0$ est tel que $\left|e(0) x_{0}(1)\right|<1$ et $e(0)\left\|\pi_{A}\left(x_{0}\right)\right\|_{\infty}<1$.

- $e(1)>0$ est tel que $\left|e(1) x_{1}(0)\right|,\left|e(1) x_{1}(1)\right|<1 / 2$ et $e(1)\left\|\pi_{A}\left(x_{1}\right)\right\|_{\infty}$ $<1 / 2$.

- $e(2)>0$ est tel que $\left|e(2) x_{2}(0)\right|,\left|e(2) x_{2}(1)\right|,\left|e(2) x_{2}(2)\right|<1 / 4$, et $e(2)\left\|\pi_{A}\left(x_{2}\right)\right\|_{\infty}<1 / 4$.

- $e(n)>0$ est tel que $\left|e(n) x_{n}(0)\right|,\left|e(n) x_{n}(1)\right|, \ldots,\left|e(n) x_{n}(n)\right|<1 / 2^{n}$, et $e(n)\left\|\pi_{A}\left(x_{n}\right)\right\|_{\infty}<1 / 2^{n}$.

Sur cette définition, on voit que la somme $s=e(1) x_{1}+e(2) x_{2}+e(3) x_{3}$ $+\ldots$ est, sur chaque composante, une série absolument convergente, donc 
converge pour la topologie produit dans $\mathbb{R}^{\omega}$, et que $\pi_{A}(s)$ est la somme d'une série absolument convergente dans $\ell_{\infty}$, donc converge dans ce Banach, et donc on a $\pi_{A}(s) \in \ell_{\infty}$. Finalement $s \in X$.

Ainsi pour toute suite de points de $X$, on peut trouver une combinaison linéaire infinie à coefficients strictement positifs qui soit définie et dans $X$, il n'existe donc sûrement pas de fonction linéaire $f: \mathbb{R}^{\omega} \rightarrow \mathbb{R}^{\omega}$ continue telle que $f^{-1}(X)=c_{00}$, car si on prend pour $x_{n}$ les $f\left(u_{n}\right)$ et qu'on a $f\left(u_{n}\right)=$ $x_{n} \in X$, le $e$ trouvé ci-dessus vérifie $e \notin c_{00}$ et $f(e) \in X$.

(iii) C'est une application de (i) et (ii) dans des théories où il y a des p-points, en remarquant que d'après (2.3.6), $X$ ne peut être complètement métrisable s'il n'est pas "encadrable".

\section{Références}

[B] N. Bourbaki, Eléments de mathématiques, Espaces vectoriels topologiques, Masson, Paris, 1981.

[C] P. Casevitz, Dichotomie pour les espaces de suites rélles, Fund. Math. 165 (2000), 249-284.

[J] T. Jech, Set Theory, Academic Press, New York, 1978.

[K] A. Kechris, Classical Descriptive Set Theory, Springer, New York, 1995.

[K-L-W] A. S. Kechris, A. Louveau and W. H. Woodin, The structure of $\sigma$-ideals of compact sets, Trans. Amer. Math. Soc. 301 (1987), 263-288.

[Sc] H. H. Schaefer, Banach Lattices and Positive Operators, Springer, New York, 1974.

[So] S. Solecki, Analytic ideals and their applications, Ann. Pure Appl. Logic 99 (1999), 51-72.

[T] M. Talagrand, Compacts de fonctions mesurables et filtres non mesurables, Studia Math. 67 (1980), 13-43.

SDAD - Université de Caen

Campus II, Boulevard Maréchal Juin

1, Esplanade de la Libération

BP 5186

F-14032 Caen Cedex, France

E-mail: casevitz@math.unicaen.fr

Received 17 December 1999;

in revised form 8 January 2001 